\title{
Self exciting threshold interest rates models
}

\author{
Marc Decamps*, Marc Goovaerts ${ }^{\dagger}$, Wim Schoutens ${ }^{\ddagger}$
}

February 28, 2005

\begin{abstract}
In this paper, we study a new class of tractable diffusions suitable for model's primitives of interest rates. We consider scalar diffusions with scale $s^{\prime}(x)$ and speed $m(x)$ densities discontinuous at the level $x^{*}$. We call that family of processes Self Exciting Threshold (SET) diffusions. Following Gorovoi and Linetsky (2004), we obtain semianalytical expressions for the transition density of SET (killed) diffusions. We propose several applications to interest rates modeling. We show that SET short rate processes do not generate arbitrage possibilities and we adapt the HJM procedure to forward rates with discontinuous scale density. We also extend the CEV and the shiftedlognormal Libor market models. Finally, the models are calibrated to the U.S. market. SET diffusions can also be used to model stock price, stochastic volatility, credit spread, etc.
\end{abstract}

Keywords and phrases: SETAR, state-price density, skew Brownian motion, eigenfunction expansions, interest rates, market models.

\footnotetext{
${ }^{*}$ K.U.Leuven, FETEW, Naamsestraat 69, B-3000 Leuven, e-mail: marc.decamps@econ.kuleuven.ac.be.

$\dagger$ K.U.Leuven and U.v.Amsterdam, FETEW, Naamsestraat 69, B-3000 Leuven, e-mail: marc.goovaerts@econ.kuleuven.ac.be.

${ }^{\ddagger}$ Department of Mathematics, Celestijnenlaan 200B, B-3001 Leuven, e-mail: Wim.Schoutens@wis.kuleuven.ac.be.
} 


\section{Introduction}

One-factor models assume that all the information about the term structure of interest rates can be summarized by a single state variable which is usually the short rate. The dynamic of the short-term interest rate has received considerable attention in the financial literature. Among many others, the Vasicek (1977) and the Cox-Ingersoll-Ross (1985) models define the short rate as a linear diffusion with mean reverting instantaneous drift that guarantees the stationarity of the process. The Vasicek model assumes a constant instantaneous volatility while the volatility of the CIR model vanishes rapidly when the short rate falls off in order to make zero unattainable. The Vasicek and the CIR models are very tractable as closed-form expressions exist for the transition density and the bond price. Unfortunately, these models partly fail in capturing the empirical behavior of short rate time series.

The Japanese interest rates since the Asian crisis illustrate the unadequacy of classical models. As mentioned by Goldstein and Keirstead (1997) and Gorovoi and Linetsky (2004), the Japanese short-term rate during the period 1996 - 2003 remained at a very low level, but with a rather high volatility. The Vasicek model is consistent with high volatility at low interest rate regime but the probability for the short rate to become negative is not negligible whereas the CIR model precludes negative interest rate through a low volatility near zero. The second difficulty encountered when modeling the Japanese term structure of interest rates relates to the socalled Zero Interest Rate Policy (ZIRP). In February 1999, the Bank of Japan adopted the ZIRP by providing the necessary liquidity to offer very cheap credit against the deflationary pressure. The ZIRP was abandoned in Augustus 2000 and reactivated on March 19, 2001. The changes in the policy of the Bank of Japan have resulted in a regime switching behavior of the short-term rate depending whether the ZIRP is activated to maintain the short rate near zero or deactivated to permit short rate around 0.5 percent. Goldstein and Keirstead (1997) provide a solution to this problem by imposing a reflecting or an absorbing boundary to the short rate process while Black (1995) proposes the use of a shadow rate. As explained in details in Gorovoi and Linetsky (2004), analytical expressions can be recovered by using eigenfunction expansions for both models. 
The U.S. interest rates have a similar regime switching feature depending on the level of the short rate. As mentioned in Pfann, Schotman and Tschering (1996), during the period 1979-1982 interest rates were very high and extremely volatile. They argue that the volatility of the U.S. interest rates plummets when the short rate falls below 8.5 percent. Markov switching regime models were introduced in the literature to capture this behavior. Under these models, the short rate switches between discrete regimes each of them driven by a diffusion process with distinct drift and volatility. AitSahalia (1996) criticizes such models on their time-inhomogeneous feature and argues in favor of a short rate process with bimodal transition probability, both modes corresponding to a different regime. This can be achieved through a diffusion process with highly nonlinear instantaneous drift and volatility, see Ait-Sahalia (1996).

Factor models are mostly used (e.g. by central banks) to understand the mechanisms driving the term struture of interest rates while more flexible models are needed for pricing derivatives. Libor market models consider the discrete forward (Libor) rates as model primitives rather than the short-term spot rate as in the Vasicek or the Cox-Ingersoll-Ross model or the continuously compounded forward rates as suggested by Heath, Jarrow and Morton (1992). It allows for pricing caplets (floorlets) as call (put) options with the popular Black's formula. In Black's formula, the forward Libor rates are defined as martingales with lognormal marginals under the appropriate risk-neutral forward measure. However, lognormal forward Libor rates do not allow for the volatility skew present in the Japanese but also in the U.S. Libor (and swap) markets. Andersen and Andreasen (2000) propose to model the volatility skew by means of CEV diffusion. Mercurio (2004) proposes mix shifted log-normal models for the forward rates, see also Joshi and Rebonato (2003).

In this paper, we propose a new family of tractable stochastic processes for model's primitives of interest rates. We consider the linear diffusion $X$ defined on the state space $I=\left(e_{1}, e_{2}\right)$ and we allow the scale $s^{\prime}(x)$ and speed $m(x)$ densities to be discontinuous at the level $x^{*} \in\left(e_{1}, e_{2}\right)$. In case $s^{\prime}(x)$ is continuous, the diffusion $\{X(t), t \geq 0\}$ is solution of a stochastic differential equation with two regimes

$$
d X(t)= \begin{cases}\mu_{1}(X(t)) d t+\sigma_{1}(X(t)) d W(t), & e_{1}<X(t)<x^{*} \\ \mu_{2}(X(t)) d t+\sigma_{2}(X(t)) d W(t), & x^{*} \leq X(t)<e_{2},\end{cases}
$$

where $\{W(t), t \geq 0\}$ is a standard Brownian motion, the differences $\mu_{2}\left(x^{*}\right)-$ $\mu_{1}\left(x^{*}\right)$ and $\sigma_{2}\left(x^{*}\right)-\sigma_{1}\left(x^{*}\right)$ are finite. We write for short that the process $X$ 
is a Self Excited Threshold (SET) diffusion with two regimes. In case $s^{\prime}(x)$ is discontinuous, we show that the linear diffusion is a semimartingale solution of a stochastic differential equation involving its symmetric local time. We interpret these diffusions in terms of the probability of switching between regimes at the level $x^{*}$. Following Linetsky (2004) and Gorovoi and Linetsky (2004), we obtain semi-analytical expressions for the transition density and prices of European-style contingent claims that facilitate the calibration of the models. In case no analytical expression exists, we propose stochastic representations that may help to find approximations.

The paper is organized as follows. In section 2, we recall the useful notion of local time and we define the skew Brownian motion. In section 3, we study properties of SET diffusions. We derive stochastic representations for the transition probability of (killed) SET diffusions that hold under mild assumptions. We adapt the results of Gorovoi and Linetsky (2004) to that class of diffusions and we obtain eigenfunction expansions for the transition density when the spectrum of the (killed) semi-group is discrete. In section 4, we study SET one-factor term structure models. We provide analytical results in terms of special functions for the cases of two Vasicek regimes and two CIR regimes. We interpret the resulting term structure as a time-continuous version of Self Exciting Threshold AutoRegressive (SETAR) time series model used by Pfann, Schotman and Tschering (1996).We discuss generalization to multi-factors models and to SET diffusions with discontinuous scale density. In section 5, we define SET Libor market models. We give expressions for the price of caplet and we adapt the approximation of Andersen and Andreasen (2000) for swaption price when adding stochastic trading time. In section 6 , we illustrate the convergence pattern of eigenfunction expansions and we calibrate a SET model with two Vasicek regimes to the U.S. zero-yield curve.

\section{Definitions}

In this paper, we present properties of the (killed) semi-group when speed and scale densities are discontinuous at the level $x^{*}$. We start with a brief introduction to the useful notions of local time and skew Brownian motion.

\section{$2.1 \quad$ Local time}

The notion of Local time was introduced by P. Lévy for measuring the time spent by a diffusion process in the vicinity of a point. Following Ouknine 
(1991), we can distinguish three local times at $a$ associated with the continuous semimartingale $X$ started at $x$ :

- the right-local time at $a$ denoted $L_{t}^{a_{+}}(X)$

$$
\frac{1}{2} L_{t}^{a_{+}}(X)=(X(t)-a)^{+}-(x-a)^{+}-\int_{0}^{t} 1_{(X(s)>a)} d X(s),
$$

- the left-local time at $a$ denoted $L_{t}^{a_{-}}(X)$

$$
\frac{1}{2} L_{t}^{a_{-}}(X)=(X(t)-a)^{-}-(x-a)^{-}+\int_{0}^{t} 1_{(X(s)<a)} d X(s)
$$

- the symmetric local at a denoted $L_{t}^{a}(X)$

$$
L_{t}^{a}(X)=\left(L_{t}^{a_{+}}(X)+L_{t}^{a_{-}}(X)\right) / 2
$$

where $x^{+}=\sup (0, x)$ and $x^{-}=\sup (0,-x)$. If $L_{t}^{a_{+}}(X)=L_{t}^{a_{-}}(X)$, we say that the local time is continuous at $a$. The symmetric local time satisfies the occupation formula

$$
\int_{0}^{t} \nu(X(s)) d\langle X, X\rangle_{s}=\int_{\mathbb{R}} \nu(x) L_{t}^{x}(X) d x
$$

for every bounded measurable function $\nu(x)$.

As outlined in Lejay (2002), local times play an essential role in the study of stochastic processes with non-regular coefficients. Lejay (2002) shows that processes whose generators have discontinuous coefficients are solutions of stochastic differential equations in the form

$$
d X(t)=\sigma(X(t)) d W(t)+\int_{\mathbb{R}} \nu(x) d L_{t}^{x}(X) d x
$$

where $\{W(t), t \geq 0\}$ is a standard Brownian motion. For more details on stochastic differential equations involving their local time, we refer also to Le Gall (1981). When $\sigma=1$ and $\nu(x)=(2 \alpha-1) \delta_{(x-a)}$ where $\delta_{(x-a)}$ is a Dirac function with support $\{a\}$, the solution of (3) is the skew Brownian motion. In what follows, we will extensively use the following Lemma which is a particular case of Tanaka formula. 
Lemma 1 Suppose that the functions $f: \mathbb{R} \rightarrow \mathbb{R}, f^{\prime}$ and $f^{\prime \prime}$ exist and are continuous except at a where the limits

$$
f^{\prime}\left(a_{ \pm}\right)=\lim _{x \rightarrow a_{ \pm}} f^{\prime}(x) \quad f^{\prime \prime}\left(a_{ \pm}\right)=\lim _{x \rightarrow a_{ \pm}} f^{\prime \prime}(x)
$$

are finite. Then, for any continuous semimartingale $X$,

$f(X(t))-f(X(0))=\int_{0}^{t} f^{\prime}(X(s)) d X(s)+\frac{1}{2} \int_{0}^{t} f^{\prime \prime}(X(s)) d\langle X, X\rangle_{s}+\gamma L_{t}^{a}(X)$

where $\gamma=\frac{1}{2}\left[f^{\prime}\left(a_{+}\right)-f^{\prime}\left(a_{-}\right)\right]$.

Proof. The proof is an application of Tanaka formula as $f$ is the difference of two convex functions, see Revuz and Yor (1998).

\subsection{Skew Brownian motion}

The skew Brownian motion (skew BM) was first mentioned by Itô and McKean (1974). Since then many authors have been interested by this diffusion process. We cite Walsh (1978), Harrison and Shepp (1981), Le Gall (1982) and Ouknine (1991). A skew Brownian motion with parameter $0 \leq \beta \leq 1$ behaves like a Brownian motion away from the origin and is reflected to the positive side with probability $\beta$ and to the negative side with probability $1-\beta$ when it arrives at the origin. It can be defined as a solution of the stochastic differential equation

$$
d X^{\beta}(t)=d W(t)+(2 \beta-1) d L_{t}^{0}\left(X^{\beta}\right)
$$

where $\{W(t), t \geq 0\}$ is a standard Brownian motion. The skew BM is a piecewise linear function of a time changed Brownian motion. An application of Tanaka formula yields that the process $r_{\beta}\left(W\left(\tau_{\beta}(t)\right)\right)$ where $\tau_{\beta}(t)=\inf \left\{s \mid \int_{0}^{s} d u / \sigma_{\beta}^{2}(W(u))>t\right\}, r_{\beta}(x)=\int^{x} d y / \sigma_{\beta}(y)$ and $\sigma_{\beta}(x)=$ $(1-\beta) 1_{(x \geq 0)}+\beta 1_{(x<0)}$ is the skew BM. As shown in details by Harrison and Shepp (1981), the skew BM is a linear diffusion with discontinuous scale $s^{\prime}(x)$ and speed $m(x)$ densities defined by

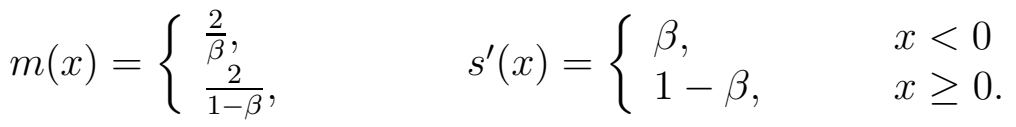


The local time of the skew Brownian motion is discontinuous at the origin. As pointed out by Ouknine (1991)

$$
\begin{aligned}
\frac{1}{2}\left(L_{t}^{0^{+}}\left(X^{\beta}\right)-L_{t}^{0^{-}}\left(X^{\beta}\right)\right) & =\int_{0}^{t} 1_{\left(X^{\beta}(s)=0\right)} d X^{\beta}(s) \\
& =\int_{0}^{t} 1_{\left(X^{\beta}(s)=0\right)} d W(s)+(2 \beta-1) L_{t}^{0}\left(X^{\beta}\right) \\
& =(2 \beta-1) L_{t}^{0}\left(X^{\beta}\right),
\end{aligned}
$$

since $L_{t}^{0}\left(X^{\beta}\right)=\frac{1}{2}\left(L_{t}^{0^{+}}\left(X^{\beta}\right)+L_{t}^{0^{-}}\left(X^{\beta}\right)\right)$, it follows that

$$
\begin{aligned}
& L_{t}^{0^{+}}\left(X^{\beta}\right)=2 \beta L_{t}^{0}\left(X^{\beta}\right) \\
& L_{t}^{0^{-}}\left(X^{\beta}\right)=2(1-\beta) L_{t}^{0}\left(X^{\beta}\right) .
\end{aligned}
$$

The transition density $p^{\beta}(t ; x, y)$ of the skew BM is obtained via its Green function

$$
p^{\beta}(t ; x, y)=\frac{1}{\sqrt{2 \pi t}} e^{-\frac{1}{2 t}|y-x|^{2}}+\operatorname{sign}(y) \frac{(2 \beta-1)}{\sqrt{2 \pi t}} e^{-\frac{1}{2 t}(|y|+|x|)^{2}},
$$

see e.g. Walsh (1978). It is also convenient to introduce the continuous semimartingale $\left\{R^{\beta}(t), t \geq 0\right\}$ with decomposition

$$
R^{\beta}(t)-R^{\beta}(0)=\int_{0}^{t} \frac{1}{R^{\beta}(s)} d s+W(t)+(2 \beta-1) L_{t}^{1}\left(R^{\beta}\right) .
$$

\section{Self Exciting Threshold (SET) diffusions}

Consider a linear conservative diffusion $X$ started at $x$ taking values in the interval $I=\left(e_{1}, e_{2}\right)$. Let $\left\{\mathcal{P}_{t}, t \geq 0\right\}$ be the semi-group of operators such that for every bounded function $f$

$$
\begin{aligned}
\left(\mathcal{P}_{t} f\right)(x) & :=E_{x}[f(X(t))] \\
& =\int_{I} f(y) p(t ; x, y) d y
\end{aligned}
$$

where $p(t ; x, y)$ is the transition density $w . r . t$. the Lebesgue measure. The scale function $s(x)$ and the speed density $m(x)$ give rise to the next representation of the infinitesimal generator of $X, \mathcal{G}: f \in \mathcal{D} \rightarrow g$ :

$$
\int_{[a, b)} g(x) m(d x)=\frac{d f}{d s}(b)-\frac{d f}{d s}(a)
$$


where $\frac{d f}{d s}(x)=\lim _{y \rightarrow x} \frac{f(y)-f(x)}{s(y)-s(x)}$ is the $s$-derivative, acting on the domain

$$
\mathcal{D}=\left\{f: f, \mathcal{G} f \in C_{b}(I), \frac{d f}{d s}(x) \text { exists, conditions at } e_{1} \text { and } e_{2}\right\} .
$$

Usual assumptions are the continuity of the functions $s, s^{\prime}, s^{\prime \prime}, m$ and $k$. As mentioned in the introduction, we consider rather that $s^{\prime}$ and $m$ are discontinuous at the level $x^{*}$ where the differences $s^{\prime}\left(x_{+}^{*}\right)-s^{\prime}\left(x_{-}^{*}\right)$ and $m\left(x_{+}^{*}\right)-m\left(x_{-}^{*}\right)$ are finite. The infinitesimal generator is the second order operator

$$
\mathcal{G} f=\frac{1}{2} \sigma^{2}(x) \frac{d^{2} f}{d x^{2}}+\mu(x) \frac{d f}{d x}
$$

where the functions $\mu$ and $\sigma$ are respectively the infinitesimal drift and volatility coefficient. The conditions on $s$ and $m$ imply that the functions $\mu$ and $\sigma$ can be discontinuous at the level $x^{*}$ where $\mu\left(x_{+}^{*}\right)-\mu\left(x_{-}^{*}\right)$ and $\sigma\left(x_{+}^{*}\right)-\sigma\left(x_{-}^{*}\right)$ are finite. In case $s^{\prime}$ is continuous, the functions $\mu$ and $\sigma$ are related to the basic characteristics $s$ and $m$ through the fomula

$$
\begin{aligned}
& s^{\prime}(x)=\exp \left\{-\int^{x} \frac{2 \mu(z)}{\sigma^{2}(z)} d z\right\} \\
& m(x)=\frac{2}{s^{\prime}(x) \sigma^{2}(x)},
\end{aligned}
$$

and, under mild assumptions, $X$ is a Itô process solution of the stochastic differential equation

$$
d X(t)=\mu(X(t)) d t+\sigma(X(t)) d W(t), \quad X(0)=x .
$$

We now show that the solution of the stochastic differential equation involving its local time

$$
d X(t)=\sigma(X(t)) d W(t)+\int_{\mathbb{R}} \nu(x) d L_{t}^{x}(X) d x, \quad X(0)=x
$$

where $\nu(x)=\mu(x) / \sigma^{2}(x)+(2 \alpha-1) \delta_{\left(x-x^{*}\right)}$, is a linear diffusion with discontinuous scale density. We define the continuous function $r_{\alpha}(x)=\int{ }^{x} r_{\alpha}^{\prime}(y) d y$ such that $r_{\alpha}\left(x^{*}\right)=x^{*}$ and $r_{\alpha}^{\prime}(x)=\frac{1}{\alpha} 1_{\left(x<x^{*}\right)}+\frac{1}{1-\alpha} 1_{\left(x \geq x^{*}\right)}$ for some $\alpha \in(0,1)$. Consider the process $\left\{Y_{t}, t \geq 0\right\}$ solution of the stochastic differential equation

$$
d Y(t)=\frac{\mu\left(r_{\alpha}(Y(t))\right)}{r_{\alpha}^{\prime}(Y(t))} d t+\frac{\sigma\left(r_{\alpha}(Y(t))\right)}{r_{\alpha}^{\prime}(Y(t))} d W(t),
$$


$Y$ is a linear diffusion with scale and speed densities given by

$$
s_{Y}^{\prime}(y)=\exp \left\{-2 \int^{r_{\alpha}(y)} \nu(z) d z\right\} .
$$

We can verify using Tanaka formula and the occupation identity (2) that $X=r_{\alpha}(Y)$. The scale and speed densities of $X$ result from the relations $s^{\prime}(x)=s_{Y}^{\prime}\left(r_{\alpha}^{-1}(x)\right) / r_{\alpha}^{\prime}\left(r_{\alpha}^{-1}(x)\right)$ and $m(x)=m_{Y}\left(r_{\alpha}^{-1}(x)\right) / r_{\alpha}^{\prime}\left(r_{\alpha}^{-1}(x)\right)$. It is easy to check that $s^{\prime}$ is discontinuous at $x^{*}$ where $(1-\alpha) s^{\prime}\left(x_{-}^{*}\right)=\alpha s^{\prime}\left(x_{+}^{*}\right)$. We write for short that $X$ is a Skew Self Exciting Threshold (SSET) diffusion with skew parameter $\alpha$. SSET diffusions have a nice interpretation in terms of the probability of switching between regimes when $X$ reaches the level $x^{*}$. Indeed, the probability of hitting $x^{*}+\epsilon$ before $x^{*}-\epsilon$ when $X$ is located at $x^{*}$ is given by

$$
\begin{aligned}
P_{x^{*}}\left(H_{x^{*}+\epsilon}<H_{x^{*}-\epsilon}\right) & =\frac{s\left(x^{*}-\epsilon\right)-s\left(x^{*}\right)}{s\left(x^{*}-\epsilon\right)-s\left(x^{*}+\epsilon\right)} \\
& =\frac{\int_{x^{*}}^{x^{*}-\epsilon} s^{\prime}(y) d y}{\int_{x^{*}}^{x^{*}-\epsilon} s^{\prime}(y) d y+\int_{x^{*}+\epsilon}^{x^{*}} s^{\prime}(y) d y}
\end{aligned}
$$

where $H_{x^{*} \pm \epsilon}=\inf \left\{t \geq 0: X(t)=x^{*} \pm \epsilon\right\}$. This probability is close to $\alpha$ for small value of $\epsilon$. We can interpret the quantity $(2 \alpha-1) \int_{0}^{t} e^{-\delta s} d L_{s}^{r^{*}}(r)$ as the $\operatorname{cost}^{1}$ of maintaining high regime of $X$ with probability $\alpha$ when it hits $x^{*}$.

The price of a claim contingent on $X$ with payout $h \in C_{b}(I)$ is the expectation under some risk neutral measure of the discounted payments. Gorovoi and Linetsky (2004) introduce the pricing semi-group $\left\{\hat{\mathcal{P}}_{t}, t \geq 0\right\}$

$$
\begin{aligned}
\left(\hat{\mathcal{P}}_{t} h\right)(x) & :=E_{x}\left[e^{-\int_{0}^{t} r(X(s)) d s} h(X(t))\right] \\
& =\int_{I} \hat{p}(t ; x, y) h(y) d y
\end{aligned}
$$

where $\hat{p}(t ; x, y)$ is called the state-price density and can be interpreted as the price of fundamental securities, or Arrow-Debreu securities that yield 1 only if $X$ equals $y$ at time to maturity. When the discount function $r($.$) takes non-negative values on I,\left\{\hat{\mathcal{P}}_{t}, t \geq 0\right\}$ is the semi-group of a linear diffusion killed at a rate $r(x)$. Let $\hat{X}$ be the non-conservative linear diffusion

\footnotetext{
${ }^{1} \delta$ is a constant discount factor.
} 
with scale and speed densities defined by (10) sent to a cemetery $\partial$ when

the additive functional $\int_{0}^{t} r(X(s)) d s$ exceeds an independent exponential random variable $\tau$ with parameter 1 , then

$$
\begin{aligned}
\left(\hat{\mathcal{P}}_{t} h\right)(x) & =E_{x}\left[h(X(t)) 1_{(\tau<t)}\right] \\
& =E_{x}[h(\hat{X}(t))]
\end{aligned}
$$

assuming that $f(\partial)=0$. The generator of $\hat{X}$ is defined by $\hat{\mathcal{G}}: f \in \mathcal{D} \rightarrow g$

$$
\int_{[a, b)} g(x) m(d x)=\frac{d f}{d s}(b)-\frac{d f}{d s}(a)-\int_{[a, b)} f(x) k(x) d x
$$

where $k(d x)=m(d x) r(x)$ is the killing measure and acts on the same domain as $\mathcal{G}$. We refer to Gorovoi and Linetsky (2004) and Linetsky (2004) for a complete account on pricing semi-groups.

\subsection{Stochastic representations}

Under some regularity conditions upon the infinitesimal drift $\mu$ and infinitesimal volatility $\sigma$, the transition density $p(t ; x, y)$ can be represented as the conditional expectation of a multiplicative functional of a Brownian motion or a three-dimensional Bessel process. These stochastic representations are widely used to prove the existence and some properties of $p(t ; x, y)$, see Dacunha-Castelle and Florens-Zmirou (1986) and Ait-Sahalia (1999) and (2002). The following Theorem generalizes this result for SET diffusions. We can also rely on these representations to derive approximations when no closed-form expression exists for $p(t ; x, y)$, see e.g. Decamps, De Schepper and Goovaerts (2004).

Theorem 1 Let $\{W(t), t \geq 0\}$ be a Brownian motion on the probability space $\left(\Omega, \mathcal{F},\left(\mathcal{F}_{t}\right)_{t \geq 0}, \mathbb{Q}\right)$ and $\{X(t), t \geq 0\}$ be the solution of the stochastic differential equation

$$
d X(t)=\mu(X(t)) d t+\sigma(X(t)) d W(t)+(2 \alpha-1) d L_{t}^{x^{*}}(X), \quad X(0)=x_{0}
$$

taking values in the interval $I_{X}$. We assume that the functions $\sigma, \sigma^{\prime}$ and $\mu$ exist and are continuous except at $x^{*} \in I_{X}$ where the limits

$$
\sigma\left(x_{ \pm}^{*}\right)=\lim _{x \rightarrow x_{ \pm}^{*}} \sigma(x), \quad \sigma^{\prime}\left(x_{ \pm}^{*}\right)=\lim _{x \rightarrow x_{ \pm}^{*}} \sigma^{\prime}(x), \quad \mu\left(x_{ \pm}^{*}\right)=\lim _{x \rightarrow x_{ \pm}^{*}} \mu(x)
$$


exist and are finite. We define the non-decreasing continuous function

$$
\phi(x)=\int^{x} \frac{d z}{\sigma(z)}
$$

such that $\phi(0)=0, y_{0}=\phi\left(x_{0}\right), y=\phi(x), y^{*}=\phi\left(x^{*}\right)$. The process $\{Y(t)=\phi(X(t)), t \geq 0\}$ is a solution of the stochastic differential equation $d Y(t)=\mu_{Y}(Y(t)) d t+d W(t)+(2 \beta-1) d L_{t}^{y^{*}}(Y), Y(0)=y_{0}$ where

$$
\begin{aligned}
\mu_{Y}(y) & =\frac{\mu\left(\phi^{-1}(y)\right)}{\sigma\left(\phi^{-1}(y)\right)}-\frac{1}{2} \sigma^{\prime}\left(\phi^{-1}(y)\right) \\
\beta & =\frac{(\alpha+1 / 2) \sigma\left(x_{-}^{*}\right)+(\alpha-1 / 2) \sigma\left(x_{+}^{*}\right)}{\sigma\left(x_{-}^{*}\right)+\sigma\left(x_{+}^{*}\right)}
\end{aligned}
$$

and takes value in the interval $I_{Y}$. Define the quantities

$$
\begin{aligned}
\lambda_{Y}(y) & =\left(\mu_{Y}^{2}(y)+\mu_{Y}^{\prime}(y)\right) / 2 \\
\gamma & =\beta \mu_{Y}\left(y_{+}^{*}\right)-(1-\beta) \mu_{Y}\left(y_{-}^{*}\right)
\end{aligned}
$$

the following representations hold

(i) when $I_{Y}=(-\infty,+\infty)$, the transition probability of $X$ can be written as

$$
\begin{aligned}
p\left(t ; x_{0}, x\right)= & \frac{e^{\int_{y_{0}}^{y} \mu(z) d z}}{\sigma(x)} p^{\beta}\left(t ; y_{0}, y\right) \\
& \times E_{y_{0}}\left[e^{-\int_{0}^{t} \lambda_{Y}\left(X^{\beta}(s)\right) d s-\gamma L_{t}^{y^{*}}\left(X^{\beta}\right)} \mid X^{\beta}(t)=y\right]
\end{aligned}
$$

where $p^{\beta}\left(t, y_{0}, y\right)$ is the transition density of the skew BM.

(ii) When $I_{Y}=(0,+\infty)$, the transition probability of $X$ can be written as

$$
\begin{aligned}
p\left(t ; x_{0}, x\right)= & \frac{e^{\int_{y_{0}}^{y} \mu(z) d z}}{\sigma(x)} \frac{y}{y_{0}} p^{\beta}\left(t ; y_{0}, y\right) \\
& \times E_{y_{0}}\left[e^{-\int_{0}^{t} \lambda_{Y}\left(R^{\beta}(s)\right) d s-\gamma^{\prime} L_{t}^{y^{*}}\left(R^{\beta}\right)} \mid R^{\beta}(t)=y\right]
\end{aligned}
$$

where $\gamma^{\prime}=\left(\gamma-(2 \beta-1) / y^{*}\right)$ and $p^{\beta}\left(t ; y_{0}, y\right)$ is the transition density of $R^{\beta}$.

Proof. A proof can be found in Appendix A. 


\subsection{Eigenfunction expansions}

Ornstein-Uhlenbeck (O-U) and square-root (CIR) processes are very popular in finance since the transition probability is known in closed-form. When analytical solutions exist for both regimes 1 and 2, we can use the spectral theory to recover tractable expressions. According to Itô and McKean (1974), the transition density w.r.t. the Lebesgue measure associated to the semi-group with infinitesimal generator $\mathcal{G}$ satisfies the partial differential equation

$$
\mathcal{G} u(t ; x)=\frac{d}{d t} u(t ; x),
$$

and can be constructed by means of an eigenfunction expansion. The eigenfunction $\varphi_{\lambda}(x)$ is the continuous solution with continuous scale derivative $\frac{d \varphi_{\lambda}}{d s}(x)$ of the Sturm-Liouville problem

$$
-(\mathcal{G} u)(x)=\lambda u(x), \quad \forall x \in I=\left(e_{1}, e_{2}\right)
$$

for some $\lambda \in \mathcal{C}$ such that $\varphi_{\lambda}(x)$ is $m$-square integrable and satisfies appropriate boundary conditions. The ordinary differential equation (13) can be solved as soon as analytical solutions exist on the intervals $\left(e_{1}, x^{*}\right]$ and $\left[x^{*}, e_{2}\right)$.

Applications of spectral theory to finance are recent. Without claiming any exhaustiveness, we refer e.g. to Lewis (1998), Lipton (2001) and (2002), Linetsky (2004) and Gorovoi and Linetsky (2004). When the spectrum of the transition density is a countable sequence $\left\{\lambda_{n}\right\}_{n \in \mathcal{N}}$, the spectral decomposition reduces to the series

$$
\hat{p}(t ; x, y)=m(y) \sum_{n=0}^{+\infty} e^{-\lambda_{n} t} \varphi_{n}(x) \varphi_{n}(y)
$$

where $\varphi_{n}(x)$ is the normalized eigenfunction associated to $\lambda_{n}$ and $m(y)$ is the speed density. In this section, we adapt the Proposition 3.3 in Gorovoi and Linetsky (2004) to the present situation. The following Theorem gives a method to obtain the eigenfunctions and the eigenvalues for the transition density and the state-price density of SET models with discrete spectrum.

Theorem 2 Assume a SSET diffusion with skew parameter $\alpha \in(0,1)$, infinitesimal volatility $\sigma(x)=\sigma_{1}(x) 1_{\left(x<x^{*}\right)}+\sigma_{2}(x) 1_{\left(x \geq x^{*}\right)}$, infinitesimal drift $\mu(x)=\mu_{1}(x) 1_{\left(x<x^{*}\right)}+\mu_{2}(x) 1_{\left(x \geq x^{*}\right)}$ and domain $I=\left(e_{1}, e_{2}\right) ;-\infty \leq e_{1}<$ 
$e_{2} \leq+\infty$. Let $\phi_{\lambda}(x)$ be the unique (to some multiplicative constant) continuous solution with continuous scale derivative $\frac{d \phi_{\lambda}}{d s}(x)$ of the $O D E$

$$
-\frac{1}{2} \sigma_{1}^{2}(x) u^{\prime \prime}(x)-\mu_{1}(x) u^{\prime}(x)+r(x) u(x)=\lambda u(r) \quad \forall r \in\left(e_{1}, x^{*}\right]
$$

such that $\int_{e_{1}}^{x^{*}}\left|\phi_{\lambda}(x)\right|^{2} m(x) d x<+\infty$ and $\phi_{\lambda}(x)$ satisfies the appropriate condition at $e_{1}$.

Let $\psi_{\lambda}(x)$ be the unique continuous solution with continuous scale derivative $\frac{d \psi_{\lambda}}{d s}(x)$ of the $O D E$

$$
-\frac{1}{2} \sigma_{2}^{2}(x) u^{\prime \prime}(x)-\mu_{2}(x) u^{\prime}(x)+r(x) u(x)=\lambda u(x) \quad \forall x \in\left[x^{*}, e_{2}\right)
$$

such that $\int_{x^{*}}^{e_{2}}\left|\psi_{\lambda}(x)\right|^{2} m(x) d x<+\infty$ and $\psi_{\lambda}(x)$ satisfies the appropriate condition at $e_{2}$.

Then, the eigenvalues $0 \leq \lambda_{1}<\lambda_{2}<\ldots$ of the Sturm-Liouville problem (13) associated to the transition probability of the diffusion $r$ killed at a rate $r(x)$ are the zeros of the Wronskian $\omega(\lambda)$

$$
\omega(\lambda):=\phi_{\lambda}\left(x^{*}\right) \frac{\psi_{\lambda}}{d s}\left(x^{*}\right)-\psi_{\lambda}\left(x^{*}\right) \frac{\phi_{\lambda}}{d s}\left(x^{*}\right)=0
$$

and the eigenfunctions $\varphi_{n}(x)$ read

$$
\varphi_{n}(x)= \begin{cases}\sqrt{\frac{\psi_{\lambda_{n}}\left(x^{*}\right)}{\Delta_{n} \phi_{\lambda_{n}}\left(x^{*}\right)}} \phi_{\lambda_{n}}(x), & e_{1}<x \leq x^{*} \\ \sqrt{\frac{\phi_{\lambda_{n}}\left(x^{*}\right)}{\Delta_{n} \psi_{\lambda_{n}}\left(x^{*}\right)}} \psi_{\lambda_{n}}(x), & x^{*} \leq x<e_{2},\end{cases}
$$

where $\Delta_{n}=\left.\frac{d \omega(\lambda)}{d \lambda}\right|_{\lambda=\lambda_{n}}$.

Proof. The proof is similar to Proposition 3.3 in Gorovoi and Linetsky (2004), see Appendix A.

\section{SET factors models}

One-factor models of term structure are based on a single state variable which is usually the short-term rate. In this section, the short rate process $\{r(t), t \geq 0\}$ is a SSET diffusion with skew parameter $\alpha \in(0,1)$ under some risk-neutral measure. Term structures driven by SSET short rate are suitable to model target zones announced by central banks and we can 
relate the skew parameter $\alpha$ to the market uncertainty concerning monetary policies. The pricing semi-group for claims contingent on $r$ with payoff $h \in C_{b}(I)$ satisfies

$$
\begin{aligned}
\left(\hat{\mathcal{P}}_{t} h\right)\left(r_{0}\right) & :=E_{r_{0}}\left[e^{-\int_{0}^{t} r(s) d s} h(r(t))\right] \\
& =\int_{I} \hat{p}\left(t ; r_{0}, r\right) h(r) d r
\end{aligned}
$$

where $\hat{p}\left(t ; r_{0}, r\right)$ is the state-price density. We can replicate any Europeanstyle contingent claims with continuous payout $c(., s)(0 \leq s<t)$ and final payoff $h($.$) by purchasing a portfolio of Arrow-Debreu securities and$ determine the price as

$$
\int_{I} \int_{0}^{t} \hat{p}\left(\tau ; r_{0}, r\right) c(r, \tau) d r d \tau+\int_{I} \hat{p}\left(t ; r_{0}, r\right) h(r) d r
$$

see e.g. Beaglehole and Tenney (1991). In particular, the payout of a zerocoupon bond with maturity $t$ is $h(r)=1$. The payout $h(r)=(P(r, t, T)-$ $K)^{+}$where $P(r, t, T)$ is the price of the zero-coupon bond, corresponds to bond options important to evaluate the popular Black's formula for caps.

Eigenfunction expansions can be obtained for the state-price density $(r(x)=x)$ but the continuity of the scale derivative $\frac{d \varphi_{n}}{d s}(x)$ implies that the eigenfunction $\varphi_{n}(x)$ has continuous derivative except at $r^{*}$ where $(1-$ $\alpha) \varphi_{n}^{\prime}\left(r_{-}^{*}\right)=\alpha \varphi_{n}^{\prime}\left(r_{+}^{*}\right)$. As a consequence, the price of the zero-coupon bond

$$
\begin{aligned}
P(r(t), t, T) & =E_{r(t)}\left[e^{-\int_{t}^{T} r(s) d s}\right] \\
& =\int_{I_{r}} \hat{p}(T-t ; r(t), y) d y
\end{aligned}
$$

when the short rate process $\{r(t)\}_{t \geq 0}$ is a SSET diffusion with skew parameter $\alpha$, has discontinuous derivative at $r^{*}$ where $(1-\alpha) \frac{d P}{d r}\left(r_{-}^{*}\right)=\alpha \frac{d P}{d r}\left(r_{+}^{*}\right)$. An application of Lemma 1 yields

$$
\begin{aligned}
d P(r(t), t, T)= & P(r(t), t, T)(r(t) d t+\sigma(t, T) d W(t)) \\
& +\left(\alpha \frac{d P}{d r}\left(r_{+}^{*}\right)-(1-\alpha) \frac{d P}{d r}\left(r_{-}^{*}\right)\right) d L_{t}^{r^{*}}(r) \\
= & P(r(t), t, T)(r(t) d t+\sigma(t, T) d W(t))
\end{aligned}
$$

where $\{W(t), t \geq 0\}$ is a Brownian motion under the risk-neutral measure. We conclude that SSET short rate processes do not generate arbitrage between the zero-coupon bonds and the savings account $\beta(t)=$ 
$\exp \left(\int_{0}^{t} r(s) d s\right)$. Although short rates with discontinuous scale density are consistent with arbitrage free requirements, the Heath-Jarrow-Morton (HJM) procedure is not directly applicable to fit the current term structure. As mentioned by Goldstein and Keirtsead (1997) in case of a reflecting boundary, the HJM constraint determines the drift of forward rates by means of the volatility $\sigma(t, T)$ and precludes terms proportional to the local time. Following Dybvig (1997) and Goldstein and Keirtsead (1997), we suggest to add a deterministic function of time $c(t)$ to the short rate in order to recover consistency with the current term structure. The resulting short rate process is $\{c(t)+r(t), t \geq 0\}$ where $r$ is a SSET diffusion and the zero-

coupon bond price is given by $e^{-\int_{t}^{T} c(s) d s} P(r(t), t, T)$. In what follows, we give analytical expressions for the spectral representation of (S)SET models in case of two Vasicek regimes and two CIR regimes.

\subsection{SET Vasicek model}

The Vasicek model (1977) defines the short rate as the Gaussian process solution of the stochastic differential equation

$$
d r(t)=\kappa(\theta-r(t)) d t+\sigma d W(t),
$$

with state space $I=(-\infty,+\infty)$. Similarly, the Self Exciting Threshold Vasicek model is driven by the short rate process solution of

$$
d r(t)= \begin{cases}\kappa_{1}\left(\theta_{1}-r(t)\right) d t+\sigma_{1} d W(t), & -\infty<r(t)<r^{*} \\ \kappa_{2}\left(\theta_{2}-r(t)\right) d t+\sigma_{2} d W(t), & r^{*} \leq r(t)<+\infty .\end{cases}
$$

The resulting process is a scalar diffusion with scale density

$$
s^{\prime}(r)= \begin{cases}e^{\frac{k_{1}\left(\theta_{1}-r\right)^{2}}{\sigma_{1}^{2}}}, & -\infty<r<r^{*} \\ e^{\frac{k_{1}\left(\theta_{1}-r\right)^{2}}{\sigma_{1}^{2}}-\frac{k_{1}\left(\theta_{1}-r^{*}\right)^{2}}{\sigma_{1}^{2}}+\frac{k_{2}\left(\theta_{2}-r^{*}\right)^{2}}{\sigma_{2}^{2}}}, & r^{*} \leq r<+\infty ;\end{cases}
$$

and speed density $m(r)=2 /\left(s^{\prime}(r) \sigma^{2}(r)\right)$ with $\sigma^{2}(r)=\sigma_{1}^{2} 1_{\left(r<r^{*}\right)}+\sigma_{2}^{2} 1_{\left(r \geq r^{*}\right)}$ discontinuous at the level $r^{*}$. A direct application of Theorem 2 leads to the next Proposition.

Proposition 1 The functions $\phi_{\lambda}(r)$ and $\psi_{\lambda}(r)$ defined in Theorem 2 corresponding the transition density of SET Vasicek models are given by

$$
\begin{aligned}
& \phi_{\lambda}(r)=e^{z_{1}^{2} / 4} D_{\nu_{1}}\left(-z_{1}\right) \\
& \psi_{\lambda}(r)=e^{z_{2}^{2} / 4} D_{\nu_{2}}\left(z_{2}\right)
\end{aligned}
$$


where $z_{1}=\frac{\sqrt{2 \kappa_{1}}}{\sigma_{1}}\left(\theta_{1}-r\right), z_{2}=\frac{\sqrt{2 \kappa_{2}}}{\sigma_{2}}\left(\theta_{2}-r\right)$ and $D_{\nu}(z)$ are the parabolic cylinder functions of parameter $\nu_{1}=\lambda / \kappa_{1}$ and $\nu_{2}=\lambda / \kappa_{2}$.

The functions $\phi_{\lambda}(r)$ and $\psi_{\lambda}(r)$ defined in Theorem 2 corresponding to state-price density of SET Vasicek models are given by

$$
\begin{aligned}
& \phi_{\lambda}(r)=e^{z_{1}^{2} / 4} D_{\mu_{1}}\left(-\left(\alpha_{1}-z_{1}\right)\right) \\
& \psi_{\lambda}(r)=e^{z_{2}^{2} / 4} D_{\mu_{2}}\left(\alpha_{2}-z_{2}\right)
\end{aligned}
$$

where $z_{1}=\frac{\sqrt{2 \kappa_{1}}}{\sigma_{1}}\left(\theta_{1}-r\right)$ and $z_{2}=\frac{\sqrt{2 \kappa_{2}}}{\sigma_{2}}\left(\theta_{2}-r\right) ; \alpha_{1}=\sigma_{1}^{2} \sqrt{2 / \kappa_{1}^{3}}$ and $\alpha_{2}=$ $\sigma_{2}^{2} \sqrt{2 / \kappa_{2}^{3}}$; and $D_{\mu}(z)$ are the parabolic cylinder functions of parameters $\mu_{1}=$ $\sigma_{1}^{2} / 2 \kappa_{1}^{3}+\left(\lambda_{1}-\theta_{1}\right) / \kappa_{1}$ and $\mu_{2}=\sigma_{2}^{2} / 2 \kappa_{2}^{3}+\left(\lambda_{2}-\theta_{2}\right) / \kappa_{2}$.

Proof. The proof is a trivial application of Theorem 2, see Appendix A.

\subsection{SET Cox-Ingersol-Ross model}

Cox, Ingersoll and Ross (1985) propose to model the short rate as the process solution of the stochastic differential equation

$$
d r(t)=\kappa(\theta-r(t)) d t+\sigma \sqrt{r(t)} d W(t)
$$

The CIR short rate remains positive if the parameters satisfy the condition $\sigma^{2} \leq 2 \kappa \theta$. Similarly, the Self Exciting Threshold CIR model is driven by the short rate process solution of

$$
d r(t)= \begin{cases}\kappa_{1}\left(\theta_{1}-r(t)\right) d t+\sigma_{1} \sqrt{r(t)} d W(t), & 0<r(t)<r^{*} \\ \kappa_{2}\left(\theta_{2}-r(t)\right) d t+\sigma_{2} \sqrt{r(t)} d W(t), & r^{*} \leq r(t)<+\infty\end{cases}
$$

The resulting process is a linear diffusion with scale density

$$
s^{\prime}(r)= \begin{cases}r^{-\frac{2 \kappa_{1} \theta_{1}}{\sigma_{1}^{2}}} e^{\frac{2 \kappa_{1} r}{\sigma_{1}^{2}}}, & 0<r<r^{*} \\ r^{-\frac{2 \kappa_{2} \theta_{2}}{\sigma_{2}^{2}} e^{\frac{2 \kappa_{2} r}{\sigma_{2}^{2}}} r^{*} \frac{2 \kappa_{2} \theta_{2}}{\sigma_{2}^{2}}-\frac{2 \kappa_{1} \theta_{1}}{\sigma_{1}^{2}} e^{\frac{2 \kappa_{2} r^{*}}{\sigma_{2}^{2}}-\frac{2 \kappa_{1} r^{*}}{\sigma_{1}^{2}}},} & r^{*} \leq r<+\infty\end{cases}
$$

and speed density $m(r)=2 /\left(s^{\prime}(r) \sigma^{2}(r)\right)$ with $\sigma^{2}(r)=\sigma_{1}^{2} r 1_{\left(r<r^{*}\right)}+\sigma_{2}^{2} r 1_{\left(r \geq r^{*}\right)}$ discontinuous at the level $r^{*}$. The state space of $r$ is the positive half line $(0,+\infty)$ if the parameters $\kappa_{1}, \theta_{1}$ and $\sigma_{1}$ satisfy the condition $\sigma_{1}^{2} \leq 2 \kappa_{1} \theta_{1}$. A direct application of Theorem 2 leads to the next Proposition. 
Proposition 2 The functions $\phi_{\lambda}(r)$ and $\psi_{\lambda}(r)$ defined in Theorem 2 corresponding to the transition density of SET Cox-Ingersoll-Ross models are given by

$$
\begin{aligned}
& \phi_{\lambda}(r)=r^{-\frac{\kappa_{1} \theta_{1}}{\sigma_{1}^{2}}} e^{\frac{\kappa_{1} r}{\sigma_{1}^{2}}} M_{k_{1}, m_{1}}\left(2 \kappa_{1} r / \sigma_{1}^{2}\right) \\
& \psi_{\lambda}(r)=r^{-\frac{\kappa_{2} \theta_{2}}{\sigma_{2}^{2}}} e^{\frac{\kappa_{2} r}{\sigma_{2}^{2}}} W_{k_{2}, m_{2}}\left(2 \kappa_{2} r / \sigma_{2}^{2}\right)
\end{aligned}
$$

where $M_{k_{1}, m_{1}}$ is the first Whittaker function of parameters $k_{1}=\frac{\lambda}{\kappa_{1}}+\frac{\kappa_{1} \theta_{1}}{\sigma_{1}^{2}}$, $m_{1}=\frac{\kappa_{1} \theta_{1}}{\sigma_{1}^{2}}-\frac{1}{2}$ and $W_{k_{2}, m_{2}}$ is the second Whittaker function of parameters $k_{2}=\frac{\lambda}{\kappa_{2}}+\frac{\kappa_{2} \theta_{2}}{\sigma_{2}^{2}}, m_{2}=\frac{\kappa_{2} \theta_{2}}{\sigma_{2}^{2}}-\frac{1}{2}$.

The functions $\phi_{\lambda}(r)$ and $\psi_{\lambda}(r)$ defined in Theorem 2 corresponding to the state-price density of SET Cox-Ingersoll-Ross models are given by

$$
\begin{aligned}
& \phi_{\lambda}(r)=r^{-\frac{\kappa_{1} \theta_{1}}{\sigma_{1}^{2}}} e^{\frac{\kappa_{1} r}{\sigma_{1}^{2}}} M_{k_{3}, m_{1}}\left(2 \kappa_{1} r / \sigma_{1}^{2}\right) \\
& \psi_{\lambda}(r)=r^{-\frac{\kappa_{2} \theta_{2}}{\sigma_{2}^{2}}} e^{\frac{\kappa_{2} r}{\sigma_{2}^{2}}} W_{k_{4}, m_{2}}\left(2 \kappa_{2} r / \sigma_{2}^{2}\right)
\end{aligned}
$$

$k_{3}=\frac{\lambda}{\gamma_{1}}+\frac{\beta_{1} \kappa_{1}}{2 \gamma_{1}}$ and $k_{4}=\frac{\lambda}{\gamma_{2}}+\frac{\beta_{2} \kappa_{2}}{2 \gamma_{2}}$ with $\gamma_{1}=\sqrt{\kappa_{1}^{2}+2 \sigma_{1}^{2}}$ and $\gamma_{2}=\sqrt{\kappa_{2}^{2}+2 \sigma_{2}^{2}}$.

Proof. The proof is a trivial application of Theorem 2, see Appendix A.

\subsection{Multi-factor models}

It is straightforward to extend our analysis to multi-factor models. If we define the short-term interest rate process $r$ as a sum of independent (S)SET diffusions

$$
r(t)=\sum_{i=1}^{n} X^{(i)}(t),
$$

the price of the zero-coupon bond can be decomposed into the product

$$
\begin{aligned}
P(r(t), t, T) & =E_{r(t)}\left[e^{-\int_{t}^{T} r(s) d s}\right] \\
& =\prod_{i=1}^{n} E_{X^{(i)}(t)}\left[e^{-\int_{t}^{T} X^{(i)}(s) d s}\right] .
\end{aligned}
$$

Hence, a closed-form expression for bond price can be obtained as long as eigenfunction expansion exists for each factor. 


\section{$5 \quad$ SET Libor market models}

Libor market models consider the discrete forward (Libor) rates as model primitives rather than the continuously compounded forward rates as suggested by Heath, Jarrow and Morton (1992) or the short-term spot rate as in the Vasicek (1977) or the Cox-Ingersoll-Ross model (1985). In recent papers, Brace et al.(1997), Jamshidian (1997) and Miltersen et al.(1997) were able to derive simultanously (under the same forward measure) the dynamic consistent with arbitrage-free requirements of all relevant forward rates.

Assume that the zero-coupon bond price processes $\{P(t, T), 0 \leq t \leq T\}$ satisfy the stochastic differential equation

$$
\frac{d P(t, T)}{P(t, T)}=r(t) d t+\sigma(t, T) d W(t)
$$

where $\{W(t), t \geq 0\}$ is a Brownian motion under the risk-neutral measure $\mathbb{Q}$ and $\sigma(t, T)$ is the adapted volatility process. Following Musiela (1995), we define the (risk-neutral) forward measure $\mathbb{Q}_{T}$ by means of the absolute continuity relation

$$
\begin{aligned}
\mathbb{Q}_{T} & =\exp \left(-\int_{0}^{T} \sigma(t, T) d W(t)-\frac{1}{2} \int_{0}^{T} \sigma^{2}(t, T) d t\right) \mathbb{Q} \\
& =(\beta(T) P(0, T))^{-1} \mathbb{Q}
\end{aligned}
$$

where $\beta(t)=\exp \left(\int_{0}^{t} r(s) d s\right)$ is the savings account. We can verify that the process $\{P(t, s) / P(t, T), 0 \leq t \leq s\}$ is a martingale under $\mathbb{Q}_{T}$. Moreover, an application of Girsanov theorem, see e.g. Musiela (1995) and Brace et al.(1997), provides that the process

$$
W_{T}(t)=W(t)+\int_{0}^{t} \sigma(s, T) d s
$$

is a $\mathbb{Q}_{T}$-Brownian motion.

In the tenor structure $0=T_{0}<T_{1}<\ldots<T_{N+1}$, the discrete forward Libor rates are defined by the formula

$$
L_{n}(t)=\frac{1}{\delta_{n}}\left(\frac{P\left(t, T_{n}\right)}{P\left(t, T_{n+1}\right)}-1\right), \quad \delta_{n}=T_{n+1}-T_{n}
$$

for $n=0,1, \ldots, N$. By standard use of Itô formula, see e.g. De Jong, Driessen and Pelsser (2002), we obtain the following volatility structure, 


$$
\begin{aligned}
d L_{n}(t)=\ldots d t+ & L_{n}(t) \gamma_{n}(t) d W(t) \text { with } \\
\gamma_{n}(t) & =\frac{P\left(t, T_{n}\right)}{P\left(t, T_{n}\right)-P\left(t, T_{n+1}\right)}\left(\sigma\left(t, T_{n}\right)-\sigma\left(t, T_{n+1}\right)\right) \\
& =\frac{1+\delta_{n} L_{n}(t)}{\delta_{n} L_{n}(t)}\left(\sigma\left(t, T_{n}\right)-\sigma\left(t, T_{n+1}\right)\right) .
\end{aligned}
$$

Note that relation (26) is invariant under changes of measure. Since $\frac{P\left(t, T_{n}\right)}{P\left(t, T_{n+1}\right)}$ is a martingale under the risk-neutral forward measure $\mathbb{Q}_{T_{n+1}}$, we deduce that $L_{n}(t)$ is also a $\mathbb{Q}_{T_{n+1}}$-martingale. Andersen and Andreasen (2000) specify the forward rate dynamics as the solution of the stochastic differential equation

$$
d L_{n}(t)=\tilde{\sigma}\left(L_{n}(t)\right) \gamma_{n}(t) d W_{T_{n+1}}(t)
$$

for some regular function $\tilde{\sigma}$. Using relations (26) and (24), we deduce the dynamic of the forward rate $L_{n}(t)$ under the measure $\mathbb{Q}_{T_{n}}$

$$
\begin{aligned}
d W_{T_{n+1}}(t) & =d W_{T_{n}}(t)+\left(\sigma\left(t, T_{n}\right)-\sigma\left(t, T_{n+1}\right)\right) d t \\
& =d W_{T_{n}}(t)+\frac{\delta_{n} L_{n}(t)}{1+\delta_{n} L_{n}(t)} d t
\end{aligned}
$$

and, thus

$$
d L_{n}(t)=\frac{\delta_{n} \tilde{\sigma}\left(L_{n}(t)\right) \gamma_{n}(t)}{1+\delta_{n} L_{n}(t)} d t+\tilde{\sigma}\left(L_{n}(t)\right) \gamma_{n}(t) d W_{T_{n}}(t) .
$$

The payoff $\delta_{n}\left(L_{n}\left(T_{n}\right)-\kappa\right)_{+}$of a caplet offers protection against high interest rate at future time $T_{n}$. The arbitrage-free price at time $t<T_{n}$ of this security satisfies

$$
\begin{aligned}
C_{n}(t) & =E^{\mathbb{Q}}\left[\frac{\beta(t)}{\beta\left(T_{n+1}\right)} \delta_{n}\left(L_{n}\left(T_{n}\right)-\kappa\right)_{+}\right] \\
& =\delta_{n} P\left(t, T_{n+1}\right) E^{n+1}\left[\left(L_{n}\left(T_{n}\right)-\kappa\right)_{+}\right]
\end{aligned}
$$

where $E^{n+1}$ denotes the expectation relative to the forward measure $\mathbb{Q}_{T_{n+1}}$. When the forward rate $L_{n}(t)$ is lognormal as suggested in Brace et al.(1997), Jamshidian (1997) and Miltersen et al.(1997), the pricing formula (29) corresponds to the market standard Black's formula. The following proposition provides caplet price for more general instantaneous volatility function $\tilde{\sigma}$ and slighty extends Theorem 2 in Andersen and Andreasen (2000). 
Proposition 3 The process $L_{n}(\tau)$ where $\tau(t, T)=\inf \{s, v(t, s)>T\}$ with $v(t, s)=\int_{t}^{s} \gamma_{n}^{2}(u) d u$, is a scalar diffusion with scale function $s(x)=x$ and speed density $m(x)=\frac{2}{\tilde{\sigma}^{2}(x)}$. The price of a caplet with maturity $T_{n}$ and strike price $\kappa$ is given by

$$
C_{n}(t)=\delta_{n} P\left(t, T_{n+1}\right) \int_{\kappa}^{+\infty} p\left(v\left(t, T_{n}\right) ; L_{n}(t), y\right)(y-\kappa) d y
$$

where $p(t ; x, y)$ is the transition density w.r.t Lebesgue measure of $L_{n}(\tau)$.

Andersen and Andreasen (2000) provide a complete study for the case $\tilde{\sigma}(x)=x^{\beta}$. The CEV model is attractive because it allows for implied volatility skew, see Derman and Kani (1996), and offers closed-form expression for the price of caplets. Andersen and Andreasen (2000) demonstrate that the CEV model improves the fit to observed caplets and swaptions prices. Nevertheless, some drawbacks might discourage practitioners from using this model. Computing the CEV option price formula is not a trivial task as the solution involves an infinite series expansion and the numerical evaluation of an improper integral. Several approximations are proposed in the literature to overcome this difficulty, we cite e.g. Schroder (1989) and Lo, Yuen and Hui (2000). Mercurio (2004) proposes to mix shifted lognormal models with volatility $\tilde{\sigma}(x)=x-\gamma$, see also Joshi and Rebonato (2003). Those models offer tractable expressions for caplets price and flexible volatility skew. In this paper, we propose the following shifted CEV volatility

$$
\tilde{\sigma}(x)= \begin{cases}\sigma_{1}\left(x-\alpha_{1}\right)^{\beta_{1}}, & x<x^{*} \\ \sigma_{2}\left(x-\alpha_{2}\right)^{\beta_{2}}, & x \geq x^{*},\end{cases}
$$

for some constants $\sigma_{1}, \sigma_{2}, \beta_{1}, \beta_{2}, \alpha_{2}, l>0$ and $\alpha_{1}>l$. Roughly speaking, when the time changed forward rates cross the threshold $x^{*}$, the instantaneous volatility makes a jump of magnitude $\left|\sigma_{2}\left(x^{*}-\alpha_{2}\right)^{\beta_{2}}-\sigma_{1}\left(x^{*}-\alpha_{1}\right)^{\beta_{1}}\right|$. The process $L_{n}(\tau)$ is a scalar diffusion on $\left(\alpha_{1},+\infty\right)$ with scale function $s(x)=x$ and discontinuous speed measure $m(x)=2 / \tilde{\sigma}^{2}(x)$. We rely on standard results about scalar diffusion for the boundary classification, see e.g. Davydov and Linetsky (2001).

\subsection{SET lognormal Libor model}

When $\beta_{1}=1$ and $\beta_{2}=1$, the time changed forward Libor rate $L_{n}(\tau)$ is a SET diffusion with scale function $s(x)=x$ and speed density $m(x)=\frac{2}{\tilde{\sigma}^{2}(x)}$ 
where $\tilde{\sigma}(x)=1_{\left(x<x^{*}\right)} \sigma\left(x-\alpha_{1}\right)+1_{\left(x \geq x^{*}\right)} \sigma\left(x-\alpha_{2}\right)$. The spectrum is purely continuous of multiplicity two, however, closed-form expression exists for its transition density.

Proposition 4 Define $\phi(x)=\int^{x} \frac{d z}{\tilde{\sigma}(z)}$ such that $\phi\left(x^{*}\right)=0$, the transition density of $L_{n}(\tau)$ is equal to $p\left(t ; x_{0}, x\right)=p_{Y}\left(t ; \phi\left(x_{0}\right), \phi(x)\right) / \tilde{\sigma}(y)$ where

$$
\begin{aligned}
& \exp \left(-\frac{1}{2}\left(y-y_{0}\right)+\frac{1}{8} t\right) p_{Y}\left(t ; y_{0}, y\right) \\
& = \begin{cases}p^{\beta}\left(t ; y_{0}, y\right)-(1-\beta) \gamma e^{\gamma k} e^{\frac{1}{2} \gamma^{2} t} \operatorname{erfc}\left(\gamma \sqrt{t / 2}+\frac{k}{\sqrt{2 t}}\right), & y<0 \\
p^{\beta}\left(t ; y_{0}, y\right)-\beta \gamma e^{\gamma k} e^{\frac{1}{2} \gamma^{2} t} \operatorname{erfc}\left(\gamma \sqrt{t / 2}+\frac{k}{\sqrt{2 t}}\right), & y \geq 0,\end{cases}
\end{aligned}
$$

with $k=|y|+\left|y_{0}\right|, p^{\beta}\left(t ; y_{0}, y\right)$ is the transition density of the skew Brownian motion, $\gamma=\frac{(2 \beta-1)}{2}$ and $\beta=\tilde{\sigma}\left(x_{-}^{*}\right) / \tilde{\sigma}\left(x_{-}^{*}\right)+\tilde{\sigma}\left(x_{+}^{*}\right)$.

\subsection{Constant Elasticity of Variance SET Libor models}

In case $\beta_{1}<1$ and $\beta_{2}>1$ the time changed forward Libor rate $L_{n}(\tau)$ is a SET diffusion with purely discrete spectrum. The spectral representation of its transition density reduces to the series expansion given in the next Proposition.

Proposition 5 The functions $\phi_{\lambda}(r)$ and $\psi_{\lambda}(r)$ defined in Theorem 2 corresponding to the transition density of the $S E T$ diffusion $L_{n}(\tau)$ are given by

$$
\begin{aligned}
& \phi_{\lambda}(x)=\sqrt{x+\alpha_{1}} J_{\gamma_{1}}\left(\sqrt{2 \lambda} z_{1}\right) \\
& \psi_{\lambda}(x)=\sqrt{x+\alpha_{2}} Y_{\gamma_{2}}\left(\sqrt{2 \lambda} z_{2}\right)
\end{aligned}
$$

where $J_{\gamma}(x)$ is a Bessel function of first kind with parameter $\gamma_{1}=\frac{1}{2\left|\beta_{1}-1\right|}$ and $z_{1}=\frac{2 \alpha_{1}}{\sigma_{1}}\left(x+\alpha_{1}\right)^{\beta_{1}-1}$ and $Y_{\gamma}(x)$ is a Bessel function of second kind with parameter $\gamma_{2}=\frac{1}{2\left|\beta_{2}-1\right|}$ and $z_{2}=\frac{2 \alpha_{2}}{\sigma_{2}}\left(x+\alpha_{2}\right)^{\beta_{2}-1}$.

Proof. The proof is a trivial application of Theorem 2, see Appendix A.

\subsection{Adding stochastic trading time}

Joshi and Rebonato (2003) propose a stochastic volatility extension of the (shifted) log-normal Libor market. Under those models, the coefficients of 
the parametrized volatility function $\gamma_{n}(t)$ are driven by Ornstein-Uhlenbeck processes. The spectral representation (14) separates the time and space dependencies of the transition density $p(t ; x, y)$ and enables efficient implementation of stochastic volatility by random changes in time just as Carr and $\mathrm{Wu}$ (2004) for option pricing under Lévy models. Assume that the square root $(\mathrm{CIR})$ process $\{\mathrm{a}(t), t \geq 0\}$ solution of the SDE

$$
d \mathrm{a}(t)=\kappa(\theta-\mathrm{a}(t)) d t+\sigma \sqrt{\mathrm{a}(t)} d W(t), \quad \mathrm{a}(0)=a
$$

models the instantaneous activity rate in the market. We specify the forward rate dynamics by means of the following SDE

$$
d L_{n}(t)=\tilde{\sigma}\left(L_{n}(t)\right) \gamma_{n}^{*}(t) d W_{T_{n+1}}(t)
$$

where $\gamma_{n}^{*}(t)=\gamma_{n}(t) \sqrt{\mathrm{a}(t)}$. Following e.g. Carr and $\mathrm{Wu}$ (2004), the continuous and increasing process $v^{*}(t)=\int_{0}^{t} \gamma_{n}^{*^{2}}(u) d u$ defines a random clock and the forward rates taken at $\tau^{*}(t)=\inf \left\{s, v^{*}(s)>t\right\}$ satisfies

$$
d L_{n}(\tau(t))=\tilde{\sigma}\left(L_{n}(\tau(t))\right) d W_{T_{n+1}}^{*}(t)
$$

where $\left\{W_{T_{n+1}}^{*}(t), t \geq 0\right\}$ is a $\mathbb{Q}_{T_{n+1}}$-Brownian motion. In the simple case where $W$ is independent of $W_{T_{n+1}}$, the transition density of the forward rate $L_{n}(t)$ can be easily expressed using the spectral representation (14):

$$
p_{L_{n}}(t ; x, y)=m(y) \sum_{n=0}^{+\infty} E_{a}\left[e^{-\lambda_{n} \int_{0}^{t} \gamma_{n}^{2}(u) \mathbf{a}(u) d u}\right] \varphi_{n}(x) \varphi_{n}(y)
$$

The expectation is nothing else but the price of a zero-coupon bond under a simple extended square root interest rate model, see Jamshidian (1995). For the sake of completeness, we recall that

$$
E_{a}\left[e^{-\lambda \int_{t}^{T} \gamma_{n}^{2}(u) \mathbf{a}(u) d u}\right]=\exp \left(-\lambda \int_{t}^{T} B_{T}(s) \kappa \theta \gamma_{n}^{2}(s) d s-B_{T}(t)\right)
$$

where $B_{T}(t)$ satisfies

$$
B_{T}^{\prime}(t)=\lambda\left(\kappa \gamma_{n}^{2}(t)-2 \gamma_{n}(t) \gamma_{n}^{\prime}(t)\right) B_{T}(t)+\frac{1}{2} \sigma^{2} \lambda^{2} \gamma_{n}^{4}(t) B_{T}^{2}(t)-1
$$

and $B_{T}(t)=0$, we refer to Jamshidian (1995) for a complete account on that class of tractable square root models. 
The stochastic volatility Libor rate dynamic (31) is as tractable as the deterministic model starting from the spectral representation of $L_{n}\left(\tau^{*}\right)$. In order to check the consistency of Libor and swap markets, we also need narrow approximations for the price of swaptions. In what follows, we show that the method proposed by Andersen and Andreasen (2000) still applies. A swap contract is the agreement to exchange the floating interest rate payment $\delta_{j} L_{j}\left(T_{j}\right)$ with the fixed amount $\delta_{j} \theta$ between the start-date $T_{s}$ and end-date $T_{e}(s \leq j<e \leq N+1)$. The holder of a swaption has the right to enter a swap agreement at maturity date $T_{s}$. Using Black's formula for swap, we deduce the payoff generated by a swaption at maturity $T_{s}$

$$
\left(1-P\left(T_{s}, T_{e}\right)-\theta \sum_{j=s}^{e-1} P\left(T_{s}, T_{j+1}\right)\right)_{+} .
$$

Define the swap rate $R$ as the value of $\theta$ that makes the value of the underlying swap equal to zero,

$$
R(t)=\frac{P\left(t, T_{s}\right)-P\left(t, T_{e}\right)}{C(t)}
$$

where $C(t)$ is the accrual factor $C(t)=\delta_{s} P\left(t, T_{s+1}\right)+\ldots+\delta_{e-1} P\left(t, T_{e}\right)$, the payoff (33) can written in a form that suggests $C(t)$ as numéraire

$$
C\left(T_{s}\right)\left(R\left(T_{s}\right)-\theta\right)_{+} \cdot
$$

Under the measure $\mathbb{Q}_{S}$ induced by that choice of numéraire, the swap rate is a martingale driven by the SDE

$$
d R(t)=\sum_{j=s}^{e-1} \frac{\partial R(t)}{\partial L_{j}(t)} d L_{j}(t) .
$$

We now assume as suggested by Andersen and Andreasen (2000) that the ratio $\tilde{\sigma}\left(L_{j}(t)\right) / \tilde{\sigma}(R(t))$ is constant and we make the following approximation

$$
\begin{aligned}
d R(u) & \approx \tilde{\sigma}(R(u)) \sqrt{\mathrm{a}(u)} \sum_{j=s}^{e-1} \omega_{j}(t) \gamma_{j}(u) d W_{\mathbb{Q}_{S}}(u) \\
& =\tilde{\sigma}(R(u)) \gamma_{R}^{*}(u) d W_{\mathbb{Q}_{S}}(u), \quad u \geq t
\end{aligned}
$$

where

$$
\begin{aligned}
\omega_{j}(t) & =\frac{\partial R(t)}{\partial L_{j}(t)} \frac{\tilde{\sigma}\left(L_{j}(t)\right)}{\tilde{\sigma}(R(t))} \\
\gamma_{R}^{*}(u) & =\sqrt{\mathrm{a}(u)} \sum_{j=s}^{e-1} \omega_{j}(t) \gamma_{j}(u), \quad u \geq t
\end{aligned}
$$


and $\left\{W_{\mathbb{Q}_{S}}(t), t \geq 0\right\}$ is a Brownian motion under the measure $\mathbb{Q}_{S}$. Equivalently, the time-change process $R\left(\tau^{*}\right)$ where $\tau^{*}(t)=\inf \left\{s, \int_{0}^{s} \gamma_{R}^{*^{2}}(u) d u>t\right\}$ is a linear diffusion with scale function $s(x)=x$ and speed density $m(x)=$ $2 / \tilde{\sigma}^{2}(x)$. The risk-neutral price of a swaption

$$
S(t)=C(t) E^{\mathbb{Q}_{S}}\left[\left(R\left(T_{s}\right)-\theta\right)_{+}\right]
$$

can then easily be approximated using the spectral representation of the transition density of $R\left(\tau^{*}\right)$.

\section{Assessing SET interest rates models}

\subsection{Numerical illustrations}

In this section, we illustrate the convergence of the series expansions for the SET vasicek and SET Cox-Ingersoll-Ross models. We use Matlab together with the routines from the Web page http://ceta.mit.edu/comp_spec_func to evaluate the special functions. Figure 1 presents the series expansions with $k$ terms for the transition probability of a SET diffusion with two Vasicek regimes. With $k=15$ terms we obtain an accurate approximation. Figure 1 clearly illustrates the bimodal feature of SET diffusions. The bimodal behavior is a consequence of the split up mean reverting effect.

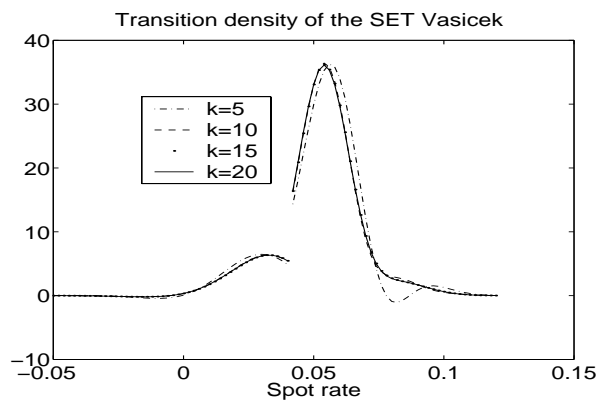

Figure 1: Eigenfunctions expansions with $k$ terms of the transition density for the SET Vasicek, $\kappa_{1}=0.25, \theta_{1}=0.03$ and $\sigma_{1}=0.015 ; \kappa_{2}=0.3, \theta_{2}=0.07$ and $\sigma_{2}=0.01$; $r^{*}=0.045$ and $t=3$.

Figure 2 plots the price of the zero-coupon bond as a function of the time to maturity together with the corresponding yield curve. We observe that the convergence pattern of eigenfunction expansions is opposite to Monte 
Carlo simulation. As the time to maturity decreases, more terms in the series have to be added to obtain the same accuracy. For maturities shorter than 2 years much more than 20 terms are needed. A solution to this numerical problem is provided in Decamps, De Schepper and Goovaerts (2004) and is based on the stochastic representations derived in section 3.1 and on the theory of comonotonic risks, see Dhaene et al. (2002).
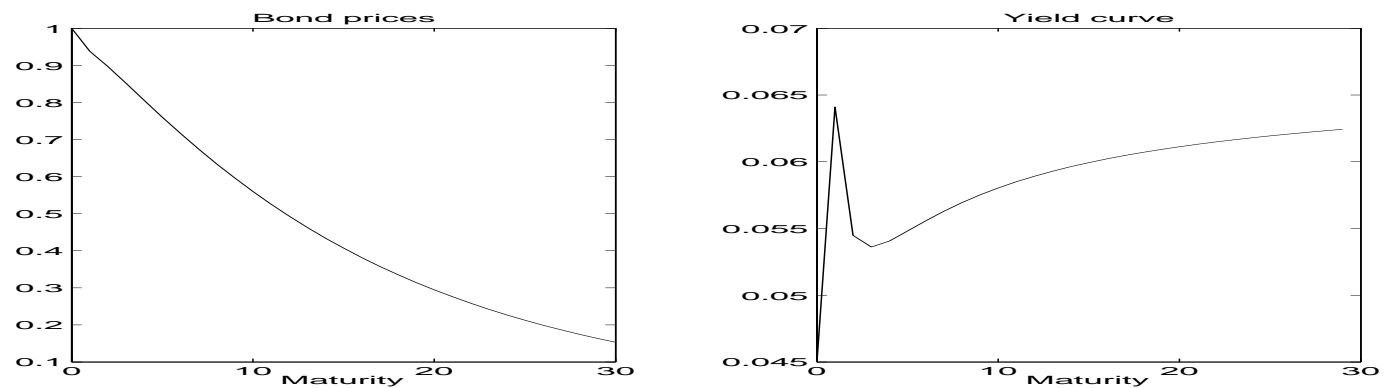

Figure 2: Eigenfunctions expansions with 20 terms of the term structure for the SET Vasicek, $\kappa_{1}=0.25, \theta_{1}=0.03$ and $\sigma_{1}=0.015 ; \kappa_{2}=0.3, \theta_{2}=0.07$ and $\sigma_{2}=0.01$; $r=0.045$

Finally, figure 3 illustrates the convergence of series expansions for the transition probability of a SET diffusion with two CIR regimes.

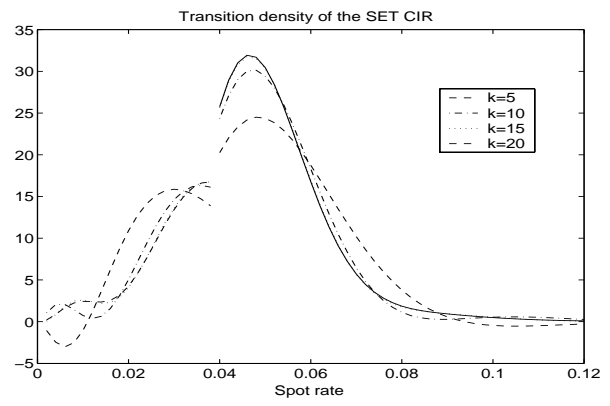

Figure 3: Eigenfunctions expansions with $k$ terms of the transition density for the SET Vasicek, $\kappa_{1}=0.12, \theta_{1}=0.03$ and $\sigma_{1}=0.05 ; \kappa_{2}=0.1, \theta_{2}=0.06$ and $\sigma_{2}=0.04 ; r^{*}=0.04$ and $t=3$. 


\subsection{Calibration of SET factor models}

In this section, we calibrate a SET Vasicek model to the U.S. bond market. The data set consists of 15 STRIPS bond prices obtained from Datastream on $14 / 12 / 2003$. We minimize the root squared error between the STRIPS yield curve and the model yield curve ${ }^{2}$. The optimization procedure provides the parameter estimates $\kappa_{1}=0.3999, \theta_{1}=0.0606$ and $\sigma_{1}=0.0105$; $\kappa_{2}=0.197, \theta_{2}=0.097$ and $\sigma_{2}=0.0284 ; r^{*}=0.0813$ for the SET Vasicek and $\kappa=0.2563, \theta=0.0654$ and $\sigma=5.119 e^{-5}$ for the Vasicek model. Figure 4 compares the STRIPS yield curve with the Vasicek and the SET Vasicek yield curves (with $k=120$ terms). The SET vasicek model improves significantly the fit to the current term structure. The volatility estimate of the Vasicek model is almost zero which is consistent with low levels of the U.S. short-term rate rate but in contradiction with higher regimes. Figure 5 plots the state-price density of the SET Vasicek and illustrates the contribution of each regime to the bond price. Finally, we draw the same conclusions than Pfann, Schotman and Tschering (1996), the U.S. short-term rate have two distinct regimes with a discontinuity of the volatility around 8.5 percent.

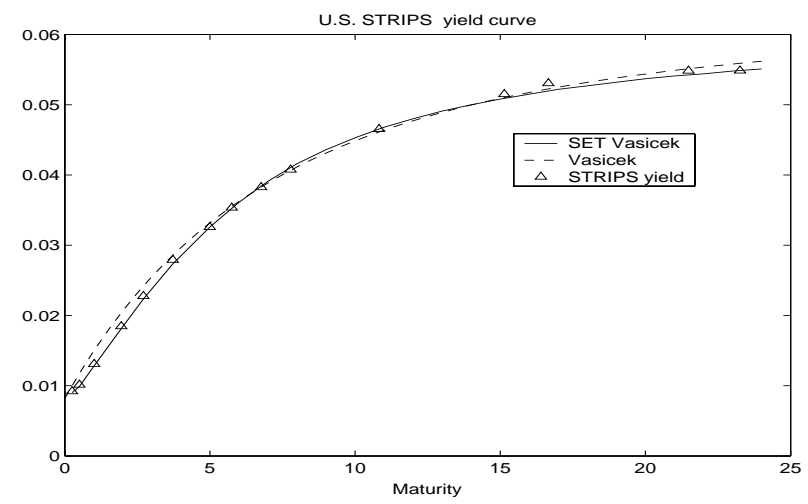

Figure 4: U.S. zero-yield curve on 14/12/2003, Datastream

\section{Acknowledgements}

Marc Decamps and Marc Goovaerts wish to thank the "Onderzoeksraad K.U.Leuven" for a GOA grant, Wim Schoutens thanks the Fund for Scientific Research - Flanders (Belgium) (F.W.O.) for a Postdoctoral Fellow.

\footnotetext{
${ }^{2}$ We only consider bonds with maturities larger than $2 \mathrm{Y}$ to avoid numerical problems
} 

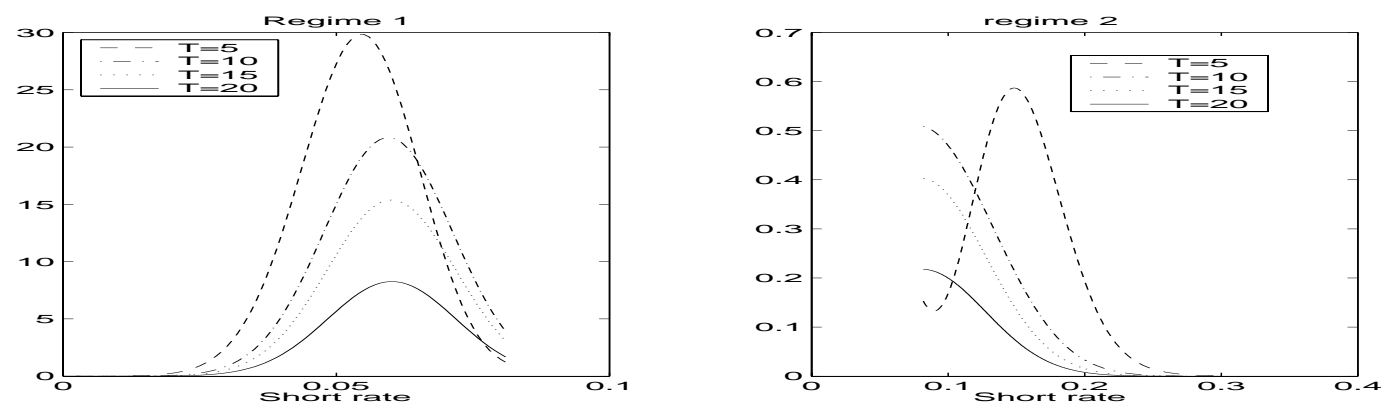

Figure 5: Regimes of the SET Vasicek model, regime 1 on $(-\infty, 0.0813]$ and regime 2 on $(0.0813,+\infty)$

\section{Corresponding author:}

Marc Decamps

Katholieke Universiteit Leuven, Department of Applied Economics

Naamsestraat 69, 3000 Leuven, Belgium

Tel: +32 16326770

e-mail: marc.decamps@econ.kuleuven.ac.be

\section{A Proofs}

\section{A.1 Proof of Theorem 1}

We start by transforming the process $\{X(t), t \geq 0\}$ into a unit volatility process $\{Y(t)=\phi(r(t)), t \geq 0\}$. Applying Lemma 1 to the process $Y$, we obtain that

$$
\begin{aligned}
d Y(t)= & \left(\frac{\mu(X(t))}{\sigma(X(t))}-\frac{1}{2} \sigma^{\prime}(X(t))\right) d t+d W(t) \\
& +\left(\frac{1}{2}\left(\frac{\sigma\left(x_{-}^{*}\right)-\sigma\left(x_{+}^{*}\right)}{\sigma\left(x_{-}^{*}\right) \sigma\left(x_{+}^{*}\right)}\right)+(2 \alpha-1)\left(\frac{\sigma\left(x_{-}^{*}\right)+\sigma\left(x_{+}^{*}\right)}{\sigma\left(x_{-}^{*}\right) \sigma\left(x_{+}^{*}\right)}\right)\right) d L_{t}^{x^{*}}(X) .
\end{aligned}
$$

If we define $\mu_{Y}(y)=\frac{\mu\left(\phi^{-1}(y)\right)}{\sigma\left(\phi^{-1}(y)\right)}-\frac{1}{2} \sigma^{\prime}\left(\phi^{-1}(y)\right)$, as $L_{t}^{y^{*}}(Y)$ satisfies

$$
\begin{aligned}
L_{t}^{y^{*}}(Y) & =\frac{1}{2}\left(L_{t}^{y_{+}^{*}}(Y)+L_{t}^{y_{-}^{*}}(Y)\right) \\
& =\frac{L_{t}^{x_{+}^{*}}(X)}{2 \sigma\left(x_{+}^{*}\right)}+\frac{L_{t}^{x_{-}^{*}}(X)}{2 \sigma\left(x_{-}^{*}\right)}
\end{aligned}
$$




$$
=\frac{\sigma\left(x_{+}^{*}\right)+\sigma\left(x_{-}^{*}\right)}{2 \sigma\left(x_{+}^{*}\right) \sigma\left(x_{-}^{*}\right)} L_{t}^{x^{*}}(X)
$$

we finally obtain that $Y$ is a solution of the stochastic differential equation

$$
d Y(t)=\mu_{Y}(Y(t)) d t+d W(t)+(2 \beta-1) d L_{t}^{y^{*}}(Y)
$$

where $L_{t}^{y^{*}}(Y)$ is the symmetric local time of $Y$.

Step 1 - We first consider the case where $I_{Y}=(-\infty,+\infty)$. For every set $A \in \mathcal{B}\left(I_{X}\right)$

$$
\begin{aligned}
P_{A}\left(x_{0}, t\right) & =E_{x_{0}}\left[1_{(X(t) \in A)}\right] \\
& =E_{y_{0}}\left[1_{\left(\phi^{-1}(Y(t)) \in A\right)}\right] .
\end{aligned}
$$

We define the measure $\mathbb{P}$ by the Radon-Nikodym derivative

$$
Z(t):=\frac{d \mathbb{Q}}{d \mathbb{P}}(t)=e^{-\frac{1}{2} \int_{0}^{t} \mu_{Y}^{2}(Y(s)) d s+\int_{0}^{t} \mu_{Y}(Y(s)) d W(s)} .
$$

From Girsanov's Theorem, $Y$ is a skew BM under the measure $\mathbb{P}$, thus

$$
E_{y_{0}}\left[1_{(Y(t) \in B)}\right]=E_{y_{0}}\left[1_{\left(X^{\beta}(t) \in B\right)} Z(t)\right]
$$

where the set $B=\phi(A) \in \mathcal{B}\left(I_{Y}\right)$ and $Z$ satisfies, according to Lemma 1 , the relation

$$
\begin{aligned}
Z(t) & =e^{-\frac{1}{2} \int_{0}^{t} \mu_{Y}^{2}\left(X^{\beta}(s)\right) d s+\int_{0}^{t} \mu_{Y}\left(X^{\beta}(s)\right) d W(s)} \\
& =e^{-\frac{1}{2} \int_{0}^{t} \mu_{Y}^{2}\left(X^{\beta}(s)\right) d s+\int_{0}^{t} \mu_{Y}\left(X^{\beta}(s)\right) d X^{\beta}(s)-(2 \beta-1)\left(\frac{\mu_{Y}\left(y_{+}^{*}\right)+\mu_{Y}\left(y_{-}^{*}\right)}{2}\right) L_{t}^{y^{*}}\left(X^{\beta}\right)} \\
& =e^{\int_{X^{\beta}(0)}^{X^{\beta}(t)} \mu_{Y}(z) d z} e^{-\int_{0}^{t} \lambda_{Y}\left(X^{\beta}(s)\right) d s-\gamma L_{t}^{y^{*}}\left(X^{\beta}\right)} .
\end{aligned}
$$

If we define $\Phi\left(t ; y_{0}, y\right)=E_{y_{0}}\left[Z(t) \mid X^{\beta}(t)=y\right]$, then

$$
E_{y_{0}}\left[1_{(Y(t) \in B)}\right]=\int_{I} 1_{(y \in B)} \Phi\left(t ; y_{0}, y\right) p^{\beta}\left(t ; y_{0}, y\right) d y
$$

By identification with equation (12), we obtain the following representation for the transition density of the process $Y$

$$
\begin{aligned}
p_{Y}\left(t ; y_{0}, y\right)= & e^{\int_{y_{0}}^{y} \mu(z) d z} p^{\beta}\left(t ; y_{0}, y\right) \\
& \times E_{y_{0}}\left[e^{-\int_{0}^{t} \lambda_{Y}\left(X^{\beta}(s)\right) d s-\gamma L_{t}^{y^{*}}\left(X^{\beta}\right)} \mid X^{\beta}(t)=y\right] .
\end{aligned}
$$

We finally deduce the expression for the transition density of $X$ through the formula

$$
p\left(t ; x_{0}, x\right)=p_{Y}\left(t ; \phi\left(x_{0}\right), \phi(x)\right) \phi^{\prime}(x) .
$$


Step 2 - We briefly discuss the case where $I_{Y}=(0,+\infty)$. If we decompose the regular part of the drift of $Y$ into the sum $\mu_{Y}(y)=\left(\mu_{Y}(y)-\frac{1}{y}\right)+\frac{1}{y}$, we can reduce the process $Y$ to the process $R^{\beta}$ using Girsanov's Theorem. We obtain the results with similar calculations as for the case $I=$ $(-\infty,+\infty)$ using Lemma 1 and the decomposition

$$
d W(t)=-\frac{1}{R^{\beta}(t)} d t-(2 \beta-1) d L_{t}^{y^{*}}\left(R^{\beta}\right)+d R^{\beta}(t) .
$$

\section{A.2 Proof of Theorem 2}

Following Lemma 1 in Linetsky (2004), it exists a unique (to some multiplicative constant) continuous solution $\eta_{\lambda_{n}}(x)$ with continuous scale derivative to the $O D E$

$$
-\frac{1}{2} \sigma^{2}(x) u^{\prime \prime}(x)-\mu(x) u^{\prime}(x)+r(x) u(x)=\lambda_{n} u(x)
$$

on the interval $I=\left(e_{1}, e_{2}\right)$ which is $m$-square integrable in a neighborhood of $e_{1}$ and satisfies the appropriate condition at the boundary $e_{1}$. As the eigenfunction $\varphi_{n}(x)$ is also $m$-square integrable in a neighborhood of $e_{1}$ and satisfies the appropriate condition at the boundary $e_{1}, \varphi_{n}(x)$ must be equal to $\eta_{\lambda_{n}}(x)$ up to a constant. Similarly, we can deduce that $\varphi_{n}(x)$ is also equal to $\xi_{\lambda_{n}}(x)$ (up to a constant) where $\xi_{\lambda_{n}}(x)$ is the unique solution of the Sturm Liouville equation (36) that is $m$-square integrable in a neighborhood of $e_{2}$ and satisfies the appropriate condition at the boundary $e_{2}$. We conclude that it exists a non-zero constant $A_{n}$ such that $\eta_{\lambda_{n}}(x)=A_{n} \xi_{\lambda_{n}}(x)$. The Wronskian is defined by

$$
\omega(\lambda):=\eta_{\lambda}(x) \frac{\xi_{\lambda}}{d s}(x)-\xi_{\lambda}(x) \frac{\eta_{\lambda}}{d s}(x)
$$

it is easy to check that $\omega(\lambda)$ depends only on $\lambda$ as $\eta_{\lambda}(x)$ and $\xi_{\lambda}(x)$ are both continuous solutions of $-(\mathcal{G} u)=\lambda u$. Moreover, $\eta_{\lambda_{n}}(x)=A_{n} \xi_{\lambda_{n}}(x)$ implies that $w(\lambda)=0$ for $\lambda=\lambda_{n}$. From Theorem 5 in Linetsky (2004), we know that $\left\|\eta_{\lambda_{n}}(x)\right\|=A_{n} \omega^{\prime}\left(\lambda_{n}\right)$ and thus, $\varphi_{n}(x)$ is continuous at $x^{*}$ and $\left\|\varphi_{n}(x)\right\|=1$. Finally we observe that $\eta_{\lambda_{n}}(x)$ is equal to $\phi_{\lambda_{n}}(x)$ on the interval $\left(e_{1}, x^{*}\right]$ and that $\xi_{\lambda_{n}}(x)$ is equal to $\psi_{\lambda_{n}}(x)$ on the interval $\left[x^{*}, e_{2}\right)$. 


\section{A.3 Proof of Proposition 1}

The function $\phi_{\lambda}(r)$ of Theorem 2 for the transition density is solution of the $O D E$

$$
-\frac{1}{2} \sigma_{1}^{2} u^{\prime \prime}-\kappa_{1}\left(\theta_{1}-r\right) u^{\prime}=\lambda u, \quad r \in(-\infty, r *]
$$

We look for solutions in the form $u(r)=e^{z_{1}^{2} / 4} v\left(z_{1}\right)$ with $z_{1}=\frac{\sqrt{2 \kappa_{1}}}{\sigma_{1}}\left(\theta_{1}-r\right)$. Substituting $u(r)$ in equation (37), we obtain that $v(z)$ satisfies the WeberHermite equation

$$
v^{\prime \prime}+\left(\frac{1}{2}+\nu_{1}-\frac{z^{2}}{4}\right) v=0, \quad z \in\left[\sqrt{2 \kappa_{1}}\left(\theta_{1}-r^{*}\right) / \sigma_{1},+\infty\right)
$$

with $\nu_{1}=\lambda / \kappa_{1}$. The solution $m$-square integrable in a neighborhood of $+\infty$ is the parabolic cylinder function $D_{\nu_{1}}\left(z_{1}\right)$. With similar arguments, we find that $\psi_{\lambda}(r)=e^{z_{2}^{2} / 4} D_{\nu_{2}}\left(-z_{2}\right)$ is the unique solution of

$$
-\frac{1}{2} \sigma_{2}^{2} u^{\prime \prime}-\kappa_{2}\left(\theta_{2}-r\right) u^{\prime}=\lambda u, \quad r \in[r *,+\infty)
$$

that is $m$-square integrable in a neighborhood of $+\infty$.

The function $\phi_{\lambda}(r)$ of Theorem 2 for the state-price density is solution of the $O D E$

$$
-\frac{1}{2} \sigma_{1}^{2} u^{\prime \prime}-\kappa_{1}\left(\theta_{1}-r\right) u^{\prime}+r u=\lambda u, \quad r \in(-\infty, r *] .
$$

We look for solutions in the form $u(r)=e^{z_{1}^{2} / 4} v\left(z_{1}\right)$ with $z_{1}=\frac{\sqrt{2 \kappa_{1}}}{\sigma_{1}}\left(\theta_{1}-r\right)$. Substituting $u(r)$ in equation (38), we obtain that $v(z)$ satisfies the WeberHermite equation

$$
v^{\prime \prime}+\left(\frac{1}{2}+\mu_{1}-\frac{\left(\alpha_{1}-z^{2}\right)}{4}\right) v=0, \quad z \in\left[\sqrt{2 \kappa_{1}}\left(\theta_{1}-r^{*}\right) / \sigma_{1},+\infty\right)
$$

with $\mu_{1}=\sigma_{1}^{2} / 2 \kappa_{1}^{3}+\left(\lambda_{1}-\theta_{1}\right) / \kappa_{1}$ and $\alpha_{1}=\sigma_{1}^{2} \sqrt{2 / \kappa_{1}^{3}}$. The solution $m$-square integrable in a neighborhood of $+\infty$ is the parabolic cylinder function $D_{\mu_{1}}(-$ $\left.\left(\alpha_{1}-z_{1}\right)\right)$. With similar arguments, we find that $\psi_{\lambda}(r)=e^{z_{2}^{2} / 4} D_{\mu_{2}}\left(\alpha_{2}-z_{2}\right)$ is the unique solution of

$$
-\frac{1}{2} \sigma_{2}^{2} u^{\prime \prime}-\kappa_{2}\left(\theta_{2}-r\right) u^{\prime}+r u=\lambda u, \quad r \in[r *,+\infty)
$$

that is $m$-square integrable in a neighborhood of $+\infty$. 


\section{A.4 Proof of Proposition 2}

The function $\phi_{\lambda}(r)$ of Theorem 2 for the transition density is solution of the $O D E$

$$
-\frac{1}{2} \sigma_{1}^{2} r u^{\prime \prime}-\kappa_{1}\left(\theta_{1}-r\right) u^{\prime}=\lambda u, \quad r \in(0, r *] .
$$

We look for solutions in the form $u(r)=r^{-\frac{\kappa_{1} \theta_{1} r}{\sigma_{1}^{2}}} e^{\frac{\kappa_{1} r}{\sigma_{1}^{2}}} v\left(z_{1}\right)$ where $z_{1}=$ $2 \kappa_{1} r / \sigma_{1}^{2}$. Substituting $u(r)$ in equation (39), we obtain that $v(z)$ satisfies the Whittaker equation

$$
v^{\prime \prime}+\left(-\frac{1}{4}+\frac{k_{1}}{z_{1}}-\frac{\frac{1}{4}-m_{1}^{2}}{z_{1}^{2}}\right) v=0, \quad z \in\left(0,2 \kappa_{1} r^{*} / \sigma_{1}^{2}\right]
$$

The solution $m$-square integrable in a neighborhood of 0 is the Whittaker function $M_{k_{1}, m_{1}}(z)$. With similar arguments, we find that $\psi_{\lambda}(r)=$ $r^{-\frac{\kappa_{2} \theta_{2}}{\sigma_{2}^{2}}} e^{\frac{\kappa_{2} r}{\sigma_{2}^{2}}}$

$W_{k_{2}, m_{2}}\left(2 \kappa_{2} r / \sigma_{2}^{2}\right)$ is the unique solution of

$$
-\frac{1}{2} \sigma_{2}^{2} r u^{\prime \prime}-\kappa_{2}\left(\theta_{2}-r\right) u^{\prime}=\lambda u, \quad r \in\left[r^{*},+\infty\right)
$$

that is $m$-square integrable in a neighborhood of $+\infty$.

The function $\phi_{\lambda}(r)$ of Theorem 2 for the state-price density is solution of the $O D E$

$$
-\frac{1}{2} \sigma_{1}^{2} r u^{\prime \prime}-\kappa_{1}\left(\theta_{1}-r\right) u^{\prime}+r u=\lambda u, \quad r \in(0, r *] .
$$

We look for solutions in the form $u(r)=r^{-\frac{\kappa_{1} \theta_{1} r}{\sigma_{1}^{2}}} e^{\frac{\kappa_{1} r}{\sigma_{1}^{2}}} v\left(z_{1}\right)$ where $z_{1}=$ $2 \gamma_{1} r / \sigma_{1}^{2}$. Substituting $u(r)$ in equation (40), we obtain that $v(z)$ satisfies the Whittaker equation

$$
v^{\prime \prime}+\left(-\frac{1}{4}+\frac{k_{3}}{z_{1}}+\frac{\frac{1}{4}-m_{1}^{2}}{z_{1}^{2}}\right) v=0, \quad z \in\left(0,2 \kappa_{1} r^{*} / \sigma_{1}^{2}\right]
$$

The solution $m$-square integrable in a neighborhood of 0 is the Whittaker function $M_{k_{3}, m_{1}}(z)$. With similar arguments, we find that $\psi_{\lambda}(r)=$ $r^{-\frac{\kappa_{2} \theta_{2}}{\sigma_{2}^{2}}} e^{\frac{\kappa_{2} r}{\sigma_{2}^{2}}}$

$W_{k_{4}, m_{2}}\left(2 \kappa_{2} r / \sigma_{2}^{2}\right)$ is the unique solution of

$$
-\frac{1}{2} \sigma_{2}^{2} r u^{\prime \prime}-\kappa_{2}\left(\theta_{2}-r\right) u^{\prime}+r u=\lambda u, \quad r \in\left[r^{*},+\infty\right)
$$

that is $m$-square integrable in a neighborhood of $+\infty$. 


\section{A.5 Proof of Proposition 4}

The proof is a direct application of Theorem (3.1). The transformed process $Y=\phi(X)$ is a solution of the stochastic differential equation

$$
d Y(t)=-\frac{1}{2} d t+d W(t)+(2 \beta-1) d L_{t}^{0}(Y), \quad Y(0)=\phi\left(x_{0}\right)=y_{0} .
$$

According to Theorem (3.1), the transition density of $Y$ satisfies

$$
e^{-\frac{1}{2}\left(y-y_{0}\right)+\frac{1}{8} t} p_{Y}\left(t ; y_{0}, y\right)=p^{\beta}\left(t ; y_{0}, y\right) E_{y_{0}}\left[e^{-\gamma L_{t}^{0}\left(X^{\beta}\right)} \mid X^{\beta}(t)=y\right] .
$$

The r.h.s. expression of the previous relation is precisely the transition density of the skew Brownian motion elastically killed at 0 computed in Decamps, De Schepper and Goovaerts (2004).

\section{A.6 Proof of Proposition 5}

The function $\phi_{\lambda}(x)$ of Theorem 2 for the transition density of $L_{n}(\tau)$ is solution of the $O D E$

$$
-\frac{1}{2} \sigma_{1}^{2}\left(x-\alpha_{1}\right)^{2 \beta_{1}} u^{\prime \prime}=\lambda u, \quad x \in(0, x *] .
$$

We look for solutions in the form $u(x)=\sqrt{x+\alpha_{1}} v\left(\sqrt{2 \lambda} z_{1}\right)$ with $z_{1}=$ $\frac{2 \alpha_{1}}{\sigma_{1}}\left(x+\alpha_{1}\right)^{\beta_{1}-1}$, we obtain that $v(z)$ satisfies the Bessel equation

$$
z^{2} v^{\prime \prime}+s v^{\prime}-\left(\gamma_{1}^{2}-2 \lambda z^{2}\right)=0, \quad z \in\left(0, \frac{2 \alpha_{1}}{\sigma_{1}}\left(x^{*}+\alpha_{1}\right)^{\beta_{1}-1}\right]
$$

with parameter $\gamma_{1}=\frac{1}{2\left|\beta_{1}-1\right|}$. The solution $m$-square integrable in a neighborhood of 0 is the Bessel funcion of first kind $J_{\gamma_{1}}(\sqrt{2 \lambda} z)$. With similar arguments, we find that $\psi_{\lambda}(x)=\sqrt{x+\alpha_{2}} Y_{\gamma_{2}}\left(\sqrt{2 \lambda} z_{1}\right)$ is the unique solution of

$$
-\frac{1}{2} \sigma_{2}^{2}\left(x-\alpha_{2}\right)^{2 \beta_{2}} u^{\prime \prime}=\lambda u, \quad x \in\left(x^{*},+\infty\right) .
$$

that is $m$-square integrable in a neighborhood of $+\infty$. 


\section{References}

[1] Ait-Sahalia Y. (1996): "Testing continuous-time models of the spot interest rate", Review of Financial Studies, vol.9, p.385-426.

[2] Ait-Sahalia Y. (1999): "Transition probabilities for interest rates and other non-linear diffusions", Journal of finance, vol.54(4), p.1361-1395.

[3] Ait-Sahalia Y. (2002): "Maximum likelihood estimation of discretely sampled diffusions: a closed-form approximation approach", Econometrica, vol.70, No. 1 (January, 2002), p.223-262.

[4] Andersen L., Andreasen J. (2000): "Volatility skews and extensions of Libor market model", Applied Mathematical Finance, vol. 7, p.1-32.

[5] Artzner F., Delbaen F. (1989): "Term structure of interest rate: the martingale approach", Advances in Applied Mathematics, vol.10, p.95129.

[6] Beaglehole D.R., Tenney M.S. (1991): "General solutions of some interest-rate claim pricing equation", Journal of fixed income, vol.2, p.69-83.

[7] Black, F. (1995): "Interest rate as options", Journal of Finance, vol .50, p.1371-1376.

[8] Brace A., Gatarek M., Musiela M. (1997): "The market model of interest rate dynamics", Mathematical Finance, vol. 7, p.127-147.

[9] Carr P., Wu L. (2004): "Time changed Lévy processes and option pricing", vol. 71, p.131-414.

[10] Cox J.C., Ingersoll J.E., Ross S.A. (1985): "A Theory of the Term Structure of Interest Rate" Econometrica, vol. 53, p.385-407.

[11] Dacunha-Castelle D., Florens-Zmirou (1986): "Estimation of coefficients of a diffusion from discrete observations", Stochastics, vol.19, p.263-284.

[12] Decamps M., De Schepper A., Goovaerts M.J. (2004): "Applications of $\delta$-function perturbations to the pricing of derivatives securities", Physica A, to appear. 
[13] De Jong F., J. Driessen, A. Pelsser (1991): "Libor versus swap market models: an empirical comparison", European Finance Review, vol. 5(3), p.201-237.

[14] Dhaene J., Denuit M., Goovearts M.J., Kaas R., Vyncke D. (2002): "The concept of comonotonicity in actuarial science and finance: theory", Insurance: Mathematics and Economics, vol. 31(1), p.3-31.

[15] Dybvig P. (1997): "Bond and bond option pricing based on the current term structure", Mathematics of Derivatives Securities, eds. Dempster M.A.H. and Pliska S., Cambridge: Cambridge University Press.

[16] Goovaerts M.J., De Schepper A., Decamps M. (2004): "Closed-form approximations for diffusion densities: a path integral approach ". Journal of Computational and Applied Mathematics, vol.165, p.337364.

[17] Gorovoi V., Linetsky V. (2004): "Black's model of interest rates as options, eigenfunction expansions and Japanese interest rate", Mathematical finance, vol.14(1), p.49-79.

[18] Itô K., McKean H.P. (1974): "Diffusion processes and their Sample Paths", Springer, Berlin.

[19] Jamshidian F. (1997): "Libor and swap market models and measures", Finance and Stochastics, vol. 1, p.293-330.

[20] Jamshidian F. (1995): "A simple class of square-root interest-rate models", Applied Mathematical Finance, vol. 2, p.61-72.

[21] Jensen B., Poulsen R. (2002): "Transition densities of diffusion processes: numerical comparison of approximation tecniques", The Journal of Derivatives, p.18-32.

[22] Joshi M., Rebonato R. (2003): "A stochastic-volatility, displaceddiffusion extension of the Libor market model", Quantitative Finance, p.458-469.

[23] Harrison J.M., Shepp L.A. (1981): "On skew Brownian motion", Ann. Probab., vol.9, p.309-313. 
[24] Le Gall J.F. (1982): "Temps locaux et equations differentielles stochastiques", Lectures Notes in Math. 986, Berlin, New-York: SpringerVerlag.

[25] Lejay A. (2003): "On the decomposition of excursions measures of processes whose generators have coefficients discontinuous at one point", Markov Processes and Related Fields, vol. 8, p.177-138.

[26] Lewis A. (1998): "Applications of eigenfunction expansion in continuous-time finance", Mathematical Finance, vol. 8, p.349-383.

[27] Linetsky V. (2004): "The spectral decomposition of the option value", International Journal of Theoretical and Applied Finance, vol.7(3), p.337-384.

[28] Lipton A. (2001): "Mathematical Methods for Foreign Exchange", World Scientific, Singapore.

[29] Lipton A. (2002): "The volatility smile problem", RISK, February, p.61-65.

[30] Mercurio F. (2004): "Pricing the smile in a forward Libor market model", Quantitative finance, to appear.

[31] Miltersen K., Sandmann K., Sondermann D. (1997): "Closed-form solutions for term structure derivatives with lognormal interest rates", Journal of Finance, vol. 52, p.409-430.

[32] Musiela M. (1995): "General framework for pricing derivatives securities", Stochastic Differential Equation,vol. 55, p.227-251.

[33] Ouknine Y. (1991): "Skew Brownian motion and derived processes", Theory of Probability and Applications, vol. 35,p.163-169.

[34] Pfann G.A., Schotman P.C. and Tschering R. (1996): "Nonlinear interest rate dynamics and implications for the term structure", Journal of Econometrics, vol. 74, p.149-176.

[35] Revuz D., Yor M. (1998): "Continuous martingales and Brownian motion", Third edition, Springer-Verlag.

[36] Vasicek O.A. (1977): "An equilibrium characterization of the term structure", Journal of Financial Economics, vol.5, p.177-188. 
[37] Walsh J. (1978): "Diffusion with discontinuous local time", Astérisque, vol.52-53, p.37-46. 